\title{
Age-related decrease in mitochondrial IncMtDloop expression potentiates Alzheimer's pathogenesis
}

\section{Wandi Xiong}

Peking University

\section{Kaiyu Xu}

Kunming Institute of Zoology, CAS

Jacquelyne SUN

Chinese University of Hong Kong

Siling Liu

Kunming Institute of Zoology, CAS

\section{Ying Zhang}

\section{Zhongyu Zhang}

Peking University

\section{Fan Liao}

Peking University

\section{Yongbo Zheng}

Peking University

\section{Jie Shi}

Peking University https://orcid.org/0000-0001-6567-8160

\section{Karl Herrup}

University of Pittsburgh School of Medicine

\section{Kim Chow}

Chinese University of Hong Kong https://orcid.org/0000-0003-1203-2096

\section{Lin Lu}

Peking University Sixth Hospital https://orcid.org/0000-0003-0742-9072

Jiali Li ( $\nabla$ jialili@bjmu.edu.cn )

Peking University https://orcid.org/0000-0001-7804-7165

\section{Article}

\section{Keywords:}

Posted Date: February 18th, 2022

DOI: https://doi.org/10.21203/rs.3.rs-1363630/v1 
License: (c) (i) This work is licensed under a Creative Commons Attribution 4.0 International License. Read Full License 


\section{Abstract}

Loss of mitochondrial homeostasis are evident in Alzheimer's disease (AD) ${ }^{1}$. However, the underlying mechanisms remain largely elusive. In this study, we report that IncMtDloop, an age-related and conserved long noncoding RNA derived from mitochondrial DNA (mtDNA) D-loop, is crucial for sustaining mitochondrial transcription and homeostasis, and associates with Alzheimer's pathogenesis. Notably, the level of IncMtDloop expression is diminished in the brains of human AD patients and the 3xTg mouse model, whereas the failure to recruit mitochondrial transcription factor A (TFAM) leads to the deregulated mitochondrial transcription. Restoring IncMtDloop expression not only significantly improves mitochondrial transcription, morphology and function, but also enhances mitophagy, ameliorates AD-like pathology, which these subcellular effects extend to a dramatic reversal of deficits in synaptic and cognitive behaviors of the 3xTg mouse model. Collectively, our findings show the decreased IncMtDloop expression potentiates $A D$ risk, thereby revealing an unexpected mitochondrial mechanism contributing to $A D$ pathogenesis and opens a new door for therapeutic intervention.

\section{Background}

Alzheimer's disease (AD) is by far the most common form of dementia in our aging societies ${ }^{2,3}$. Currently, it remains no cure or effective treatment to manage the progression of the disease ${ }^{4-6}$. Mutations on genes regulating the production of $\beta$-amyloid $(A \beta)$ plaques and tau tangles were identified in early-onset familial cases, but these could hardly explain the etiologies behind the more common but late-onset sporadic cases, which are multifactorial in nature.

Mitochondria are the major metabolic powerhouse in eukaryotic cells, generating more than $90 \%$ of cellular ATP which powers majority of cellular functions ${ }^{7,8}$. Declines in total mitochondrial quality and activities have been associated with normal aging but also the development of a wide range of agerelated disease ${ }^{9-11}$. While the relationship between nuclear genome integrity and brain aging is well recognized, the potential contribution of mitochondrial DNA (mtDNA) is also questioned ${ }^{12,13}$. The mitochondrial genome encodes 13 proteins of oxidative phosphorylation (OXPHOS) chain, which are crucial to ATP production and respiratory capacity, 2 rRNAs and 22 tRNAs, all of which are involved in the mitochondrial transcription, replication and translation in situ. The mtDNA exhibits profound influence on cellular function, including biogenesis, dynamics, metabolism and degradation ${ }^{14-16}$. Growing out from gene mutations to alternations at functional levels, dysregulated mitochondrial trafficking, biogenesis and mitophagy are now found to be associated with accelerated brain aging, neurodegeneration, and notably the pathogenesis of late-onset sporadic AD 17,18. 
Long non-coding RNAs (IncRNAs) are non-protein coding transcripts with length longer than 200 nucleotides ${ }^{19}$. They represent a class of transcriptional regulatory machinery which is also one of the fastest evolving parts of the primate genome ${ }^{20-22}$. LncRNAs may regulate gene transcription or epigenetic regulators by acting as RNA scaffolds for the assembly of chromatin- and gene-regulating higher-order complexes and to guide them to specific loci within the genome ${ }^{19,23,24}$. On the other hand, IncRNAs may serve as cis- and trans-acting modulators of protein-coding gene expression, regulators of mRNA processing, modulators of post-transcriptional control by altering mRNA stability or serving as molecular sponges or even cell signaling molecules ${ }^{25}$. In the human genome, around $40 \%$ of the known IncRNAs are uniquely expressed in the brain, which are essential part of neurogenesis, including differentiation, migration and patterning of neural progenitors; so as being crucial for effective action potential and synaptic plasticity in mature neurons ${ }^{26-28}$.

Although most of our current knowledge on IncRNAs are still those encoded from the nuclear genome, mammalian cells do express a family of long noncoding mitochondrial RNAs (LncmtRNAs). On mtDNA sequences that are complementary to genes encoding MT-ND5, MT-ND6, and Cytochrome b, three mitochondrial IncRNAs were identified where these antisense noncoding mitochondrial RNA (ASncmtRNA) expressions are being regulated by nuclear-encoded mitochondrial processing proteins ${ }^{29,30}$. On the other hand, sense noncoding mitochondrial RNA (sncmtRNA) is also reported and characterized as a 2,374 nucleotide (nt) long transcript regulating the replicative state of a cell by forming a stem-loop structure complementing with the inverted repeat of $121 \mathrm{nt}$ sequencing located at the 5 ' end of the mitochondrial 16S rRNA to regulate its stability ${ }^{31,32}$. Apart from physiological processes, IncmtRNAs are also associated with disease pathogenesis. For example, the mitochondrial long noncoding RNA uc002bqs.1, also known as Long Intergenic noncoding RNA Predicted Cardiac Remodeling (LIPCAR), is a circulating IncmtRNA serving as a biomarker for cardiac remodeling and predicts the survival of patients with cardiac failure ${ }^{33,34}$.

With deep sequencing approach, we have previously characterized the dynamic changes of global IncRNA expression in brain tissues harvested from rhesus macaque across the development and aging time span ${ }^{22}$. From there, $A C 027613.1$ is identified as an age-related and well-conserved IncRNA derived from mtDNA D-loop. In this study, we termed it IncMtDloop, and investigated its function in the regulation of mitochondrial transcription and homeostasis, as well as the contribution of its decreased expression to Alzheimer's pathogenesis.

\section{Results}

Levels of IncMtDloop expression decrease in the brains of human AD patients and the mouse model 
In our previous report, cluster analysis revealed that $A C 027613.1$ was one of the newly identified IncRNAs in rhesus macaque brain, with important relevance in brain development and aging ${ }^{22}$. In this study, extended characterization on AC027613.1 gene sequence showed that it is indeed located within the Dloop regions of mitochondrial genome (Fig. 1a), therefore we renamed it as IncMtDloop. With RNAscope in situ hybridization (RISH) using specific probe set against the mouse IncMtDloop; in combination with immunocytochemical (ICC) assays with antibody against ATP synthase F1 subunit alpha (ATP5a) serving as a specific mitochondrial marker revealed that IncMtDloop is predominantly located inside mitochondria of primary hippocampal neurons (Fig. 1b, c). To demonstrate the significance IncMtDloop in mitochondria evolution, gene comparative analysis was performed which revealed that it was a well conserved mitochondrial IncRNA among various mammalian species (Extended Data Fig. 1a). Despite size differences were identified in human (572bp), macaque (695bp) and mouse (939bp), key block sequences on IncMtDloop were evolutionally conserved, with all of them were expressed and localized in mitochondria of respective cell lines (i.e. Human SH-SY5Y, monkey Cos-7 and mouse N2A cells) of these different species (Supplementary Fig. 1a-d), as revealed by the same RISH-ICC assays mentioned above.

To confirm whether IncMtDloop is a true, novel mitochondrial IncRNA species without any protein-coding potential, we performed Phylo Codon Substitution Frequencies (PhyloCSF) analysis on the UCSC browser (Extended Data Fig. 1b). The corresponding CPC score (Extended Data Fig. 1c) and CPAT coding probability (Extended Data Fig. 1d) were almost reaching the minimum as compared to a positive control of reference coding transcripts, therefore supporting IncMtDloop has no protein-coding potentials. The secondary structure of IncMtDloop was predicted as well based on minimum free energy (MFE) (Extended Data Fig. 1e). These data revealed that IncMtDloop likely assembles a complex double-stranded stemloop, which is frequently found in other known IncRNAs. With RISH-ICC system, it also indicated that IncMtDloop is abundantly expressed in mouse primary neurons, microglia and astrocytes, hinting that it is of great importance in supporting the normal mitochondrial physiology and function in the brain (Supplementary Fig. 1e).

With the RISH assay followed by immunohistochemistry analyses (RISH-IHC), significant reductions in IncMtDloop levels were observed in the prefrontal cortex (PFC), hippocampal CA1 as well as the dentate gyrus (DG) regions of human AD samples as compared to age-and sex-matched non-demented controls (Fig. 1d, e). Similar observations were found by Northern blotting and ssRT-qPCR analyses in the same set of human brain tissue samples (Fig. 1f, j and k). These phenomena are also conserved in aged (12 months old) 3xTg mice-a triple transgenic mouse model of AD exhibits both classic A $\beta$ pathology and tauopathies with the same set of analyses (Fig. $\mathbf{~} \mathbf{g}-\mathbf{i}$, I and $\mathbf{~ m}$ ). Of note, decreases in IncMtDloop expression were differentially found in 3xTg mice multiple organs which its function linkage to mitochondrial organelle (Supplementary Fig. 1f). 
$A \beta$ cascading hypothesis is one of dominating hypotheses addressed for explaining the onset and pathogenesis of $A D^{35}$. With RISH, it revealed that levels of IncMtDloop were significantly reduced in $3 \times \mathrm{Tg}$ hippocampal neuronal culture as compared to wild type controls, so as when wild type neurons were exposed to exogenous $A \beta_{42}$ treatment (Extended Data Fig. 2a, b). The loss in IncMtDloop was further confirmed by ssRT-qPCR and northern blot analyses (Extended Data Fig. 2c-f). Mutually, restoration of InMtDloop in 3xTg hippocampal neuronal culture reduced the accumulation of A 3 (Extended Data Fig. 2g, h). As IncMtDloop is widely expressed in other cell types in the brain, as demonstrated earlier (Extended Data Fig. 2a), we then asked if their IncMtDloop expression may also respond to $A \beta$ cascade signaling stimulus. By the RISH-ICC strategy, significant reductions in IncMtDloop signals were again observed in $3 x \operatorname{Tg}$ cultured cortical neurons (Supplementary Fig. 2a, b) but not in primary microglia (Supplementary Fig. 2d, e) and astrocyte cultures (Supplementary Fig. 2g, h), as when the comparisons were made to wild type controls. Consistently, ssRT-qPCR results revealed similar patterns of observations (Supplementary Fig. 2c, $f$ and i). Collectively, these data indicated that decreased IncMtDloop expression is unique to neuronal populations and associated with $A \beta$ cascade signaling and pathology.

\section{p32 promotes the mitochondrial localization of IncMtDloop}

Previous studies indicated that IncRNA may serve as scaffolds or guides for multiple proteins and even facilitate the assembly of multiprotein complexes ${ }^{36}$. We speculated that IncMtDloop may also serve similar roles to mitochondrial proteins, therefore its potential binding partners were unbiasedly evaluated by RNA pull-down assay followed by silver staining and mass spectrometry (SS-MS) analyses (Fig. 2a). Despite our earlier data indicated that IncMtDloop is predominantly located in mitochondria, chances are some of which may also present in the cytosol region, we therefore performed analyses in both cytosolic and mitochondrial fractions enriched from hippocampal tissue lysates of wild type mice. Full-length IncMtDloop sequence conjugated with biotin probes was used as bait, upon incubation with different fractions of lysates, streptavidin-conjugated beads were used to capture the biotin-containing complexes, followed by SS-MS global analyses and western blotting as validation. Using the anti-sense IncMtDloop as negative control, the full-length sense IncMtDloop uniquely pulled down a group of interacting proteins (Supplementary Fig. 3a-c). With these candidates, large-scale prediction on protein-RNA associations and the interaction propensities between polypeptide and nucleotide chains were conducted using the catPAPID algorithm on the basis of the physical-chemical properties between the binding partners ${ }^{37-39}$. Among all uniquely pulled down proteins by the IncMtDloop, Syntaxin1B (Stx1b), ATP5d and p32 were among the ones with the highly potential interaction scores and discriminative power with IncMtDloop in mitochondrial fraction (Fig. 2b). For that in the cytosolic fraction, Aly/REF export factor (Alyref), tubulin polymerization promoting protein (Tppp) and p32 were those with the highest interaction scores and discriminative powers instead (Fig. 2c). With the mitochondrial extracts, interactions between IncMtDloop and ATP5d, as well as that with p32 but not Stx1b were confirmed by Western blotting analyses (Fig. 2d). 
Similarly, IncMtDloop interactions with non-mitochondrial proteins Alyref and Tppp were also confirmed from cytosolic extracts (Fig. 2e).

p32 is also known as SF2-associated p32, p32/TAP and gC1qR. It is a conserved eukaryotic protein that localizes in the mitochondria, despite it is also present in other subcellular compartments ${ }^{40,41}$. It is thought to be involved in mitochondrial oxidative phosphorylation regulation and nucleus-mitochondrial communication ${ }^{42,43}$. As interaction between in vitro transcribed-IncMtDloop and p32 was detected in both cytosolic and mitochondrial extracts (Fig. 2f), thus indicating high confidence of interaction. Together with the distinct discriminative power and interactive score between p32 and IncMtDloop (Fig. $\mathbf{2 g}$ ) as revealed by the catRAPID algorithm, as well as their colocalization pattern revealed by the RISHICC in primary neurons (Fig. $\mathbf{2 h}$, i), we therefore use this protein as a study model for identifying the structural motifs on IncMtDloop that are critical for mediating RNA-protein interactions. Using RNA immunoprecipitation (RIP) assay followed by Northern blotting or qPCR analyses, IncMtDloop could be efficiently precipitated by p32 proteins in extracts of hippocampus and prefrontal cortex tissues freshly harvested from 12-month-old wild type mice (Fig. 2j, k). With computational prediction analysis by catRAIPD, a number of regions located at the $5^{\prime}$ and $3^{\prime}$ terminal domains of the IncMtDloop were predicted being essential for protein-RNA interactions (Supplementary Fig. 3d, e and f). Based on this information, a series of truncated IncMtDloop were constructed and subjected to the same RIP assay (Fig. 2I, m). Among various constructs, only the one that constitutes the 3'-terminal 637-939 nt fragment revealed significant interactions with p32. While the levels of RNA transcripts of neither p32 nor IncMtDloop showed direct relationship with one another (Extended Data Fig. 3a-d), protein levels of p32 were diminished in the hippocampal tissues of $3 x \mathrm{Tg}$ mice with significance found only in aged ones (i.e. 12 months old) as compared to age-matched wild type (Extended Data Fig. 3e, f); hinting the reduced IncMtDloop level as a result of aberrant $\mathrm{Ab}$ cascade signaling might affect the p32 protein abundance potentially its coordinating roles on mitochondrial translation ${ }^{44}$, mitophagy and oxidative fuel metabolism ${ }^{41}$ in neurons. Morphological alterations of mitochondria are reported to be associated to metabolic and energy deficiency in neurons in $A D$ and other neurodegenerative disorders. Notably, mitochondrial dysfunction is also a hallmark of Ab-induced neuronal toxicity in $A D{ }^{45,46}$ and similar changes were welldocumented previously in the AD-mouse model of our choice ${ }^{47}$. Given that strong binding of IncMtDloop with p32 protein which processes a mitochondrial-targeting signal at its $\mathrm{N}$-terminus ${ }^{48}$, it is therefore possible that endogenous p32 may facilitate the transport of ectopically expressed IncMtDloop into the mitochondria by protein-RNA interaction. Using primary neurons where ectopic IncMtDloop was expressed, lentiviral particles of either carrying scrambled or p32 specific siRNA constructs were infected to these cells. Upon p32 knockdown in N2A cell line, using RNAscope and qPCR assays significant reductions in mitochondrial located ectopic IncMtDloop RNA were observed as compared to control (Extended Data Fig. 3g, h). Accordingly, p32 can serve as an ideal binding partner of ectopically expressed IncMtDloop, targeting them to mitochondrial region. 


\section{Recruitment of TFAM by IncMtDloop is indispensable for the mitochondrial transcription initiation}

Damages to mtDNA accumulate over the aging process, as also the neural mtDNA mutations can contribute to aging-associated neurodegenerative disease like $A D^{49}$. As we found reduced levels of mtDNA copy number in 12-month-old 3xTg mice (Fig. 3a), we further explore the connection between IncMtDloop and mtDNA. In mitochondrial fraction, IncMtDloop can also interact with transcription factor complex in SS-MS results, suggesting that IncMtDloop may interact with mitochondrial transcription factor as a guide to regulate mitochondrial genome integrity. mtDNA is packaged in DNA-protein complexes named nucleoids, which contain the proteins required for mtDNA transcription, replication, translation and degradation ${ }^{50}$. Within these mtDNA transcription factors, we performed a collection of prediction on protein-RNA associations using the catPAPID algorithm. Among these proteins, TFAM, a promoter-binding protein and a key role in mitochondrial transcription initiation ${ }^{51,52}$, attracted our attention for its established role in interaction scores (Fig. 3b). Consistently, we performed western blot and RIP and reconfirmed the interaction between IncMtDloop and TFAM (Fig. 3c, d), and found 1-626 nt fragment of IncMtDloop revealed significant interactions with TFAM (Fig. $3 \mathbf{e}, \mathbf{h}$ ). We found the mRNA and protein expression levels of TFAM in 12-month-old 3xTg mice without significant reduction compared with WT (Extended Data Fig.4a-c) and there was little regulatory influence of IncMtDloop in TFAM protein expression (Extended Data Fig.4e, f). Given the TFAM initiates mitochondrial transcription by binding to the HSP and LSP upstream of the transcription start site (TSS) in a reversed orientation ${ }^{53,54}$, we then tested whether IncMtDloop regulated the TFAM occupation of the promoter locus. We analyzed +200 -1000 bp locus within mitochondrial D-loop region, and found +200 -400 bp segment within the mitochondrial D-loop region is the sufficient binding site for TFAM through the luciferase reporter assays (Fig. 3g and Extended Data Fig. 4d). With chromatin immunoprecipitation (ChIP) results, we found that overexpression of IncMtDloop increase the binding ability of TFAM with promoters within the mitochondrial D-loop region (Fig. 3f). The apparent increases in mitochondrial mRNA levels were the broad effect of regulatory function of IncMtDloop (Extended Data Fig. $\mathbf{4 g}$ ). These data suggested that TFAM could be recruited to the promoters within the mitochondrial D-loop region by IncMtDloop to assemble the initiation complex, and trigger mtDNA transcription (Fig. 3i).

\section{Restoring LncMtDloop alleviates AD-related changes in mitochondrial morphology and oxidative fuel metabolism}

mtDNA encodes the core component of OXPHOS complexes that is closely related with the synthesis of cellular ATP. As we found decreased levels of mtDNA copy number in AD, these accumulated damages to mtDNA integrity can have pleiotropic effects on mitochondrial function. To test whether diminished level of IncMtDloop contributes to mitochondrial abnormalities in AD-mouse model, we adopted a reverse approach and see if replenishment of neural IncMtDloop could reverse that phenomenon. With the aged 
11-month-old 3xTg mice and their age-matched controls, adeno-associated viral particle (AAV) carrying a construct that expresses GFP-tagged IncMtDloop under the regulation of Synapsin-1 promoter (i.e. SYN: AAV9- IncMtDloop) was stereotaxically microinjected into the hippocampus region. At six weeks after the initial injection, brain tissues were harvested, sliced and observed under the microscope to validate GFPtagged IncMtDloop expression (Extended Data Fig. 4e). Next, brain tissues were then subjected to transmission electron microscopy analysis for mitochondrial ultrastructure analyses (Fig. 4a). With the images, mitochondrial ultrastructures were classified based on their number of cristae, these included Class I (>3 cristae); II (2-3 cristae) and III (no or 1 crista) (Fig. 4c, d); as well as the matrix densities including Class $A$ (Dense matrix) and B (swollen and hypodense matrix) (Fig. 4e, f). In 3xTg mice, approximately $30 \%$ of the mitochondria belong to Class III and around $40 \%$ in Class B, which means that they are mainly small, short and fragmented in morphologies. These mitochondrial cristae and matrix conditions were however improved when IncMtDloop was ectopically expressed in 3xTg neurons. The mitochondrial cristae remodeling and consequently the mitochondrial morphologies were significantly improved (Fig. 4b, d, f and g).

Mitochondria indeed exist as dynamic networks that often change shape and subcellular distribution. The number and morphology of mitochondria within a cell are controlled by precisely regulated rates of organelle fusion and fission ${ }^{55}$. We therefore speculated that ectopic re-expression of IncMtDloop might have also altered the dynamics of mitochondria. To visualize that, we performed live imaging of a new MitoTracker probe (MitoESq-635), which is an enhanced squaraine dye that could be excited at $635 \mathrm{~nm}$ and $775 \mathrm{~nm}$ the stimulated emission depletion (STED) super-resolution microscopy ${ }^{56,57}$. In $3 x \mathrm{Tg}$ neurons, mitochondrial dynamics analyses revealed abnormal patterns of fission and fusion, resulting in more abundant amounts of fragmented mitochondria than wild type neurons (Fig. 4h, Video S1 and S2). With ectopic re-expression of IncMtDLoop, this significantly improved such dynamics and mitochondrial network in 3xTg neurons (Fig. 4h, Video S3 and S4). Recent studies suggest that modelling of mitochondrial architecture by changing its dynamics is crucial for bioenergetic adaption to metabolic demands ${ }^{58}$. In line with this, mitochondrial cytochrome c oxidase activities, an enzyme that is responsible for oxygen consumption in the process of mitochondrial oxidative phosphorylation ${ }^{59}$, were found to be significantly compromised in hippocampal tissues of 3xTg mice as compared to agematched wild type controls (Fig. 4i). With ectopic expression of IncMtDloop in hippocampal tissues of $3 x \operatorname{Tg}$ mice, this treatment restored the mitochondrial cytochrome $\mathrm{c}$ oxidase activities to the level that is comparable to the control group. Further analyses on cellular oxygen consumption rate by the Seahorse Mito Stress Test revealed that ectopic expression of IncMtDloop in 3xTg primary cortical enhanced both basal and reserved respiratory capacities (Fig. 4j, $\mathbf{k}$ and I), confirming its effect in facilitating mitochondrial oxidative phosphorylation. Collectively, our results revealed that IncMtDloop played an important role in maintaining mitochondrial ultrastructure, network morphologies and metabolic functions, and that loss of which, as in part triggered by Ab cascade signaling, contributed to mitochondrial impairment found in AD. 


\section{Restoring IncMtDloop expression alleviated AD-like pathology in the brain of 3xTg mouse model}

Previous studies have proposed the presence of a feed-forward relationship between the severity of ADrelated pathologies and the degree of mitochondrial dysfunction exists ${ }^{60,61}$. With the effects found on global mitochondrial function and physiology, we speculated that these changes may well-extend to neuropathology levels. Utilizing the RISH-ICC technology, we found that after inoculation of AAV viral particle carrying vehicle construct or one that expresses IncMtDloop into hippocampal tissues, ectopic reexpression of IncMtDloop in 3xTg also resulted in significant reduction in the intensity and sizes of Ab deposits in CA1, CA3 and DG (Fig. 5a, b). ELISA assays were also performed in freshly harvested hippocampal tissues which also confirmed similar findings on Ab content (Fig. $\mathbf{5 c}$, d). In addition to Ab plaques, tangles that made up of hyperphosphorylation of tau is also a hallmark of AD. Among various different phosphorylation sites, phosphorylation at Thr231, Ser396/404, Ser202, Ser202/Thr205 and Ser212/Thr214 are considered as the ones mostly implicated in AD ${ }^{62}$. In the hippocampus of $3 \times \mathrm{Tg}$ mice, while total level of mouse Tau (TAU-5) remained unchanged among all the groups, western blot results revealed that levels of phosphorylated tau (pSer202/Thr205, pT231 and human tau) were significantly elevated in those harvested from $3 \times \mathrm{Tg}$ mice (Fig. 5e, f). Moreover, the same phenomena were observed in primary neurons and the brain of 3xTg mouse model (Supplementary Fig. 5).

Dysregulated mitochondrial trafficking, biogenesis and mitophagy are now found to be associated with accelerated brain aging, neurodegeneration, and notably the pathogenesis of late-onset sporadic $A D$ 17,18. Dysregulated mitophagy can be another conserved phenotype of $A D{ }^{63}$. To differentiate if the functional enhancing effects induced by IncMtDloop is from enhancing mitophagy or other possibilities, we first analyzed the levels of various mitophagy-associated proteins. Immunoblotting revealed that key players in mitophagy, including Parkin, PINK1, LC3B-II and ULK1 were reduced in hippocampus tissues harvested from $3 \times \mathrm{Tg}$ as compared to age-matched controls and these reductions were restored upon ectopic expression of IncMtDLoop (Fig. 5g-k). Similarly, immunofluorescence studies also revealed that signal intensities of LC3B, Parkin and PINK1 in hippocampal CA1 and CA3 regions were enhanced upon ectopic expression of IncMtDloop (Extended Data Fig. 5).

\section{Restoring IncMtDloop improves synaptic functions and reverses behavior deficits of $3 \times \mathrm{Tg}$ mouse model}

Mitochondrial dysfunction has been identified as a mechanism responsible for cognitive and memory impairment ${ }^{64,65}$. Deficits in synaptic function and cognitive impairments are also being well documented in AD-mouse model including 3xTg mice. To investigate if the beneficial effect on mitochondria as a result of re-expression IncMtDloop also lead to improvements in synaptic plasticity and cognitive decline in 3xTg mice, viral particles carrying relevant constructs were microinjected into the 
hippocampus of 11 months old of 3xTg mice. At 6 weeks after microinjection, an array of electrophysiological and behavioral tests was subsequently performed (Fig. 6a). At electrophysiological level, field excitatory postsynaptic potentials (fEPSPs) of the Schaffer collateral pathway (Fig. 6b) as well as long-term potentiation (LTP) were evaluated which revealed that the impairments found in $3 x \mathrm{Tg}$ mice could be restored upon ectopic re-expression of IncMtDloop (Fig. 6c, d). Further evaluation of on animal social, cognitive and memory-associated behavior was also performed. In a nest-building paradigm (Extended Data Fig. 6a, b) and open field paradigm (Extended Data Fig. 6c, d), 3xTg mice revealed suboptimal performance than wild type controls, but that could be rescued upon ectopic re-expression of IncMtDloop. Similarly, in a novel object recognition paradigm, 3xTg was less responsive to an introduction of a novel object than wild type controls, but this could be improved upon re-expression of IncMtDloop (Extended Data Fig. 6e). Subsequently contextual and cue fear conditioning test was also performed as it is composed hippocampus-dependent learning and memory task. Again, IncMtDloop reexpression significant improved cued freezing time compared of 3xTg mice (Fig. 6e, $f$, and Extended Data Fig. $\mathbf{6 g}$ ). Finally, to assess the effect of the IncMtDloop re-expression on the animal spatial memory, Morris water maze (MWM) test was performed. While 3xTg mice were deficient in their performance, restoration of IncMtDloop in hippocampus significantly reversed all the parameters in the MWM as compared to that of AAV9-control mice (Fig. 6g-j and Extended Data Fig. 6f). Collectively, these data confirmed that restoring IncMtDloop expression improved synaptic function and resulted in a dramatic reversal of behavioral deficits of the mouse model.

\section{Discussion}

In this study, we investigated that the loss of a well conserved, novel mitochondrial DNA D-loop derived IncRNA-IncMtDloop-is found in brain tissues of AD patients and those harvested from the 3xTg classic model of AD. With the 3xTg mouse model, we have evaluated the effect of IncMtDloop loss on neuronal mitochondrial homeostasis, and their linkages to altered cellular fuel metabolism, mitophagy, neuronal firing and behavior related to cognition and memory (Supplementary Fig. 5). LncRNAs are abundant in mammalian brains ${ }^{20,26}$, yet detailed understanding on their biological functions, particularly those uniquely located in mitochondria, remain incomplete. So far, several mtDNA derived IncRNAs have been identified in mammalian cells, these included LNCND5, LNCND6 and LNCCYTB, and they may play important roles in regulating mitochondrial gene expression and mitochondrial homeostasis ${ }^{29}$. These, unlike the more well-defined nuclear-encoded IncRNAs, the biosynthesis and functions of mitochondriaderived IncRNAs remain unclear. While we may still lack much of the mechanistic understandings regarding to how IncRNAs are being regulated, their importance on normal brain biology and physiology, and implications on neurogenerative disorders were reflected by the altered expressions in these scenarios ${ }^{66-68}$.

Our bioinformatics analyses and other experiments revealed that, IncMtDloop, as a mitochondria-located IncRNA, can mechanistically interacts with $\mathrm{p} 32$, which increase the mitochondrial location of exogenous 
IncMtDloop. Using p32 as a study model of protein-RNA interaction due to the highest interaction score predicted between this protein and IncMtDloop, we identified the 3 ' terminal region located between 627$939 \mathrm{nt}$ of IncMtDloop as the likely protein-binding motif that is sufficient and necessary for its interaction with p32. While p32 is located in multiple subcellular compartments, it appears to have a role in facilitating the mitochondrial localization of ectopically expressed IncMtDloop. This property could be utilized as a treatment strategy to re-introduce exogenous IncMtDloop into the mitochondrial when the endogenous level became diminished. This also renders a good strategy to investigate its physiological roles related to mitochondria. Classically, IncRNAs are believe to be involved in transcriptional, translational and post-translational regulations. In the mitochondria where histone proteins are absent, mutations in mtDNA appear more frequently than in nucleus. Accumulation of mtDNA mutations in nucleotides would lead to mtDNA loss, which impact cellular metabolism and extensively affect the risk of complicated late-onset brain diseases, including AD, Parkinson's disease and metabolic disease. Indeed, we found the apparent mtDNA loss in 3xTg mouse model and further identified IncMtDloop can interact with TFAM and activate mtDNA expression in cis by recruitment of TFAM to the promoters of mitochondrial D-loop region. Of note, the functional analyses of IncMtDloop showed the rescued level of mtDNA copy number,

This newly identified IncRNA is also likely to have important clinical relevance as the levels of IncMtDloop are reduced in the brains of patients with $A D$, so as in the amyloid, tau and presenilin-based $3 x \mathrm{Tg}$ mouse model. We found significant neural cell type variations, with the age- and disease-related loss of IncMtDloop being most notable in neurons rather than other cell types. In recent decades, the "mitochondrial cascade hypothesis" of AD pathogenesis has seen increasing attention. Part of the appeal of this view of $A D$ is that the nervous system is an energy-intensive organ with a high demand for the synthesis of ATP. This makes the brain particularly vulnerable to mitochondrial dysfunction. As we found IncMtDloop can rescue mtDNA loss in3xTg mice, the mitochondrial function of IncMtDloop in AD appears relatively underexplored. To bridge this gap, we used the newly developed MitoESq-635 combined with STED imaging to document the morphological consequences of IncMtDloop loss on the morphological integrity of the mitochondria in $3 x \mathrm{Tg}$ neurons. When combined with electron microscopy, this further revealed extensive mitochondrial abnormalities in cells with suboptimal level of IncMtDloop. At a more global cellular level, mitochondria network in the 3xTg neurons were found to be more fragmented, with swollen cristae and a hypodense matrix than those in wild type cells. Not surprisingly, these abnormal mitochondria are defective in fusion and fission. In $A D$, the damaged fusion and fission in mitochondrial dynamics would affect mitochondrial genome and nucleoid organization, leading to mtDNA loss and mitochondrial dysfunction ${ }^{16}$.

Unexpectedly, IncMtDloop has a substantial impact on the clearance of A $\beta$ accumulation, tauopathies and $A D$ related mitophagy in neurons. It is quite significant, therefore, that restoration of IncMtDloop 
levels alleviated virtually every behavioral and electrophysiological deficit we tested in $3 x \operatorname{Tg}$ mice. These mitigations ranged from the recovery of hippocampal LTP to restoration of deficits in hippocampaldependent learning and memory performance. Importantly, since AAV9-IncMtDloop injections were done after the symptoms of the 3xTg mice, which were already established both neuropath and behavior. This is a really important suggestion that restoration of IncMtDloop can actually reverse the course of the disease, not just prevent it.

In addition, although the causal nature of the IncRNA is clear, the exact mechanism behind the improvements needs to be worked out in more detail. It is not unexpected that deficits in energy production would degrade neurophysiological function, yet it is not immediately obvious how changes in IncMtDloop expression, either directly or indirectly would serve to regulate $A \beta$ cascade signaling. In summary, we show how an unappreciated aspect of the decreased mitochondrial IncMtDloop, the inability of it decrease in neurons to regulate mitochondrial transcription and homeostasis, contributes to Alzheimer's pathogenesis. In future, a search for small RNA molecule mimics against IncMtDloop with appropriate pharmacokinetic properties may facilitate the translation of our findings into practical clinical interventions.

\section{Methods}

Constructs, oligonucleotide and plasmids. pcDNA3.1-T7-IncMtDloop (1-939) was ordered from Genecreate company. pcDNA3.1-T7-IncMtDloop \#1 ( $\triangle$ 314-939), pcDNA3.1-T7-IncMtDloop \#2 $(\triangle 1$ 313,627-939), pcDNA3.1-T7-IncMtDloop \#3 ( $\triangle 1-626)$, pcDNA3.1-T7-IncMtDloop \#4 ( $\triangle 627-939)$, pcDNA3.1-T7-IncMtDloop \#5 ( $\triangle 1$ 1-313), pcDNA3.1-T7-IncMtDloop \#6 (314-626) were mutant from pcDNA3.1-T7-IncMtDloop (1-939). siRNA for p32 and TFAM were designed and synthesized with standard protocols. For overexpression of IncMtloop in cell lines, pSLenti-CMV-IncMtloop and pSLenti-CMV-GFP were ordered from OBiO company. For overexpression of IncMtloop in mice, pAAV9-SYN-IncMtloop and PAAV9-SYN-GFP were ordered from OBiO company. For luciferase reporter assay, the fragments of different locus of mitochondrial D-loop (+200-0, +200-200, +200-400, +200--600, +200-800, +200-1000) were cloned into pGL4.10-basic vector (Promega).

Human brain samples-Human autopsy paraffin-embedded $10 \mu \mathrm{m}$ brain sections were from the following sources with approval from the appropriate local regulatory authorities. We examined 16 case-patients graciously provided by the University of Pittsburgh Alzheimer's Disease Research Center (ADRC) brain bank with approval from the Committee for Oversight of Research and Clinical Training Involving Decedents (CORID). Each case had been diagnosed neuropathologically and ranked by Braak stage (Supplementary Table 1). Additional frozen tissue was a generous gift of the ADRC at Washington University in St. Louis (Grant P50-AG-05681) with approval from the Neuropathology Core (protocol \#T1016) at Hongkong University of Science and Technology. 


\section{Mouse line}

All mouse lines including the 3xTg line (B6. Cg-Tg (APPSwe, tauP301L)1Lfa PSEN1tm1 Mpm/J; The Jackson Laboratory) and age- and gender- matched wild-type mice controls were maintained on C57/BL6 background. Mice were group-housed in cohorts of five, separated by sex in individually home cages under specific-pathogen-free conditions with free access to food and water. The light and dark cycle was 12 hour / 12 hour and the temperature was kept constant at $23 \pm 2{ }^{\circ} \mathrm{C}$ and humidity ( $50 \pm 5 \%$ ). Mice were randomly assigned to the experimental groups. Behavioral experiments were performed at the dark cycle. Ages of mice used for each experiment were 11-13 months. All animal experiments were conducted with the licenses from Kunming Institute of Zoology, and were approved by the Animal Care and Use Committee of Kunming Institute of Zoology CAS. All animal experiments were also complied with the Biomedical Ethics Committee for animal use and protection of Peking University.

\section{Primary cells}

For primary microglia cultures, wildtype female mice or 3xTg female mice were mated with the wildtype male mice or 3xTg male from an unrelated colony. The hippocampus was isolated from newborn mice (P0-P4), and cut into small pieces. The hippocampal tissue was digested with Neurosphere Dissociation Kit (Cat\# 130-095-943) and centrifuged for 5 min at 1500rpm. The purified microglia were resuspended in DMEM/F-12 (Thermo Fisher Scientific) medium with 10\% FBS and penicillin-streptomycin (Invitrogen), and then seeded on 6- or 24-well plates with 14-21 days ${ }^{69,70}$. Cultures were kept in a $37^{\circ} \mathrm{C}$ incubator with $5 \% \mathrm{CO}_{2} .1 / 2^{\text {rd }}$ of the medium was replaced with fresh medium every 3 days. For primary astrocyte cultures, wildtype female mice or $3 x \mathrm{Tg}$ female mice were mated with the wildtype male mice or $3 x \mathrm{Tg}$ male from an unrelated colony. The hippocampus was isolated from newborn mice (P0-P4), and cut into small pieces. The hippocampal tissue was digested with Neurosphere Dissociation Kit (Cat\# 130-095943). After mechanical dissociation and centrifugation, the cells were resuspended in DMEM/F-12 (Thermo Fisher Scientific) medium with 10\% FBS, penicillin-streptomycin (Invitrogen), 15 mM HEPES, $14.3 \mathrm{mM} \mathrm{NaHCO} 3,1 \%$ Fungizone ${ }^{\circledR}$ and $0.04 \%$ gentamicin, and then seeded on 6 - or 24-well plates precoated with poly-L-lysin with $14-21$ days ${ }^{71}$. Cultures were kept in a $37^{\circ} \mathrm{C}$ incubator with $5 \% \mathrm{CO}_{2} .1 / 2^{\text {rd }}$ of the medium was replaced with fresh medium every 3 days. For primary mouse hippocampal neuronal cultures, wildtype female mice or 3xTg-AD female mice were mated with the wildtype male mice or 3xTgAD male from an unrelated colony. The hippocampus was isolated from embryos of day E17, and cut into small pieces. The hippocampal tissue was digested with Neurosphere Dissociation Kit (Cat\# 130095-943). The primary mice hippocampal neurons were resuspended in Neurobasal plus medium supplemented with B27-P (Thermo Fisher Scientific), Glutamax (Thermo Fisher Scientific) and penicillinstreptomycin (Invitrogen) and then seeded on 6- or 24-well plates pre-coated with poly-L-lysine with 14-21 days ${ }^{72}$. Cultures were kept in a $37^{\circ} \mathrm{C}$ incubator with $5 \% \mathrm{CO}_{2} .1 / 2^{\text {rd }}$ of the medium was replaced with fresh 
medium every 3 days. For the $A \beta_{1-42}$ fibrils, $A \beta_{1-42}$ peptide (sigma) was dissolved in sterile PBS, and diluted to a final concentration of $250 \mu \mathrm{M}$ and incubated on a shaker at $37^{\circ} \mathrm{C}$ for $84 \mathrm{~h}$. In DIV14 or DIV21, cultures were treated with $A \beta_{1-42}$ fibrils in indicated concentrations for $18 \mathrm{~h}$ and cells were collected for subsequent experiments ${ }^{73}$.

\section{Cell line}

N2A cells, cos-7 cells and SH-SY5Y cells were cultured in in DMEM (Thermo Fisher Scientific) medium with $10 \%$ FBS and penicillin-streptomycin (Invitrogen). Cultures were kept in a $37^{\circ} \mathrm{C}$ incubator with $5 \%$ $\mathrm{CO}_{2} .1 / 2^{\text {rd }}$ of the medium was replaced with fresh medium every 3 days.

\section{AAV microinjections in mice}

For bilateral injection of AVVs, sex-matched 11-month-old wild type and 3xTg mice were anesthetized with isoflurane. Wiping $70 \%$ ethanol were used to sterilize the surgical area, removing the skin above the skull by using aseptic techniques, and drilling small holes for the sites of injection. A volume of $0.5 \mu \mathrm{l}$ virus per hemisphere was injected at a speed of $0.075 \mu \mathrm{m} \mathrm{min}^{-1}$. The target sites for AAV injection were marked on the skull (AP $-2.0 \mathrm{~mm}, \mathrm{ML} \pm 1.5 \mathrm{~mm}$, DV $-2.0 \mathrm{~mm}$ ). After surgery, mice were housed under standardized conditions for 4-6 weeks.

\section{RNA extraction and real-time qRT-PCR}

Total RNA was extracted from cultures or mice tissues using RNAiso plus reagent following the manufacturer's instructions. RNA quantity and quality were evaluated by Nano Drop ND-1000 spectrophotometer. The real-time quantitative reverse transcription PCR (qRT-PCR) was using Reverse Transcription kit (vazyme) and SYBR Green I Master (vazyme). QPCR was performed in $10 \mu$ reaction system including $1 \mu \mathrm{l} \mathrm{cDNA}, 5 \mu \mathrm{l} 2 \times$ Master Mix, $0.1 \mu \mathrm{l}$ of forward primer $(10 \mu \mathrm{M}), 0.1 \mu \mathrm{l}$ of reverse primer $(10 \mu \mathrm{M})$, and $3.8 \mu \mathrm{l}$ of double distilled water. $\beta$-Actin was used as an endogenous reference for data normalization. Primer sequences are described in Supplementary Table. Cycle threshold values were used to calculate fold changes in gene expression using the $2^{-\Delta \Delta C t}$ method. The primer sequences of probes were seen in Supplementary Table 2.

\section{Tissue preparation and western blot}


Samples from mouse brain and primary cell culture were collected and homogenized in RIPA buffer containing protease inhibitors and phosphatase inhibitors. After centrifugation, supernatants were heated to $95^{\circ} \mathrm{C}$ for $10 \mathrm{~min}$, and resolved on $6-12 \%$ polyacrylamide precast gels. The gel was transferred to PVDF membrane at $250 \mathrm{~mA}$ for $90 \mathrm{~min}$ at $4^{\circ} \mathrm{C}$ and blocked for $1 \mathrm{hr}$ at room temperature in $5 \%$ BSA in Tris buffered saline with $0.05 \%$ Tween 20 . Membranes were incubated in primary antibody overnight at $4{ }^{\circ} \mathrm{C}$. Antibodies used for western blot were: anti-6E10 (803001, BioLegend), anti-p32 (ab24733, Abcam), antiATP5a (ab14748, Abcam), anti-LC3B (ab51520, Abcam), anti-PINK1 (ab23707, Abcam), anti-parkin (\#2132, Cell Signaling), anti-AT8 (MN1020, Thermo Fisher Scientific), anti-AT180 (MN1040, Thermo Fisher Scientific), anti-HT7 (MN1000, Thermo Fisher Scientific), anti-TAU-5 (AHB0042, Thermo Fisher Scientific), anti- $\beta$-actin (ab8227, Abcam), anti-COX IV (ab33985, Abcam), anti-Stxb1 (ab183722, Abcam), anti-Tppp (ab92305, Abcam), anti-Aly/Ref (ab202894, Abcam), anti-ATP5D (ab174438, Abcam), antiTFAM (ab252432, Abcam). All blots were imaged using HRP-conjugated secondary antibodies. After three washes for 10 min each, the fluorescence signals were quantified using Image $\mathrm{J}$ software.

\section{Northern blot}

Total RNA ( $10 \mu \mathrm{g}$ for human postmortem PFC and hippocampal frozen tissues of AD and control individuals as well as wild type and 3xTg mouse brain fresh tissues, $2 \mu \mathrm{g}$ for mouse primary neurons) was denatured using NorthernMax ${ }^{\circledR}$-Gly sample loading dye (Ambion) and resolved on $1.2 \%$ agarose gel in MOPS buffer. The gel was soaked in 1×TBE for 20 min and transferred to a Hybond-N+ membrane (GE

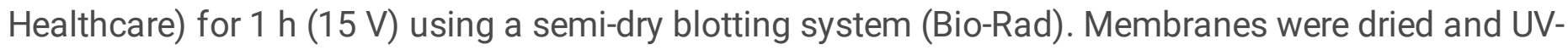
crosslinked with $150 \mathrm{~mJ} / \mathrm{cm}^{2}$ at $254 \mathrm{~nm}$. Pre-hybridization was done at $68^{\circ} \mathrm{C}$ for $1 \mathrm{~h}$, and using DIG Northern Blot Starter KIT (12039672910, Roche). DIG-labeled in vitro transcribed IncMtDLoop, and control probes were hybridized overnight. The membranes were washed three times in $2 \times$ SSC, $0.1 \%$ SDS at $68^{\circ} \mathrm{C}$ for $30 \mathrm{~min}$, followed by three $30 \mathrm{~min}$ washes in $0.2 \times \mathrm{SSC}, 0.1 \%$ SDS at $68^{\circ} \mathrm{C}$. The immunodetection was performed with anti-DIG AP-conjugated antibodies. Immunoreactive bands were visualized using CDP star reagent (Roche) and a LAS-4000 detection system (GE Healthcare). The primer sequences of probes were seen in the Supplementary Table 2.

\section{Immunofluorescence staining}

Mice were perfused with PBS and the brains were fix with 4\% paraformaldehyde (PFA). The brains were sliced into $30 \mu \mathrm{m}$ thick with a Leica CM1950 freezing microtome. For immunofluorescence staining, brain sections were permeabilized and blocked in PBS with $0.3 \%$ Triton X-100 and $10 \%$ goat serum at room temperature for 1 hour, followed by incubation with the primary antibody: anti-6E10 (803001, BioLegend), anti-p32 (ab24733, Abcam), anti-ATP5a (ab14748, Abcam), anti-LC3B (ab51520, Abcam), anti-PINK1 (ab23707, Abcam), anti-parkin (\#2132, Cell Signaling), anti-AT8 (MN1020, Thermo Fisher Scientific), antiAT180 (MN1040, Thermo Fisher Scientific), anti-HT7 (MN1000, Thermo Fisher Scientific), anti-TAU-5 
(AHB0042, Thermo Fisher Scientific). For the confocal microscopic analysis (TCS SP8, Leica), the primary antibodies were visualized with Alexa-Fluor488, Alex-Fluor594 and Alexa-Fluor647 secondary antibodies (Invitrogen). DAPI (ab104139, Abcam) was used for nuclear counter staining. For primary cultures, mice hippocampal neurons, microglia and astrocyte were fix with $4 \%$ PFA, then permeabilized and blocked in PBS with $0.3 \%$ Triton $\mathrm{X}-100$ and $10 \%$ goat serum at room temperature for 1 hour. Primary antibodies were: anti-LC3B (ab51520, Abcam), anti-PINK1 (ab23707, Abcam), anti-parkin (\#2132, Cell Signaling), antiATP5a (ab14748, Abcam), anti-lba1 (019-19741, Wako), anti-GFAP (837201, BioLegend), anti-p32 (ab24733, Abcam), anti-MAP2 (ab32454, Abcam), anti-AT8 (MN1020, Thermo Fisher Scientific), anti-HT7 (MN1000, Thermo Fisher Scientific), anti-TAU-5 (AHB0042, Thermo Fisher Scientific). For the confocal microscopic analysis (TCS SP8, Leica), the primary antibodies were visualized with Alexa-Fluor488, AlexFluor594 and Alexa-Fluor647 secondary antibodies (Invitrogen). DAPI (ab104139, Abcam) was used for nuclear counter staining.

\section{RNAscope assay for IncRNA detection}

Detection of IncMtDloop was performed on cryostat brain slices of mice and primary cultured cells. At

first, we prepared and pretreated the samples with RNAscope ${ }^{\circledR} \mathrm{H}_{2} \mathrm{O}_{2}$ and proteas reagents (Cat\# 322381). Next, we cover each slide or cells cultured on cover slips with the probe sets against human and mouse IncMtDloop (Cat\# 518381 and 518361). A positive-control probe, Homo sapiens peptidylprolyl isomerase $B(P P I B)$, and a negative-control probe for bacterial gene dap $B$ were include for each group. The probe hybridization was performed in the HybEZ ${ }^{\mathrm{TM}}$ for $2 \mathrm{~h}$ at $40^{\circ} \mathrm{C}$. The signal amplification was conducted according to the manufacturer's instruction using RNAscope ${ }^{\circledR}$ Multiplex Fluorescent Detection Reagents (Cat\# 323110). The fluorescence signals of IncMtDloop were visualized with TSA ${ }^{\circledR}$ plus fluorophore. The brain slices and cells cultured on cover slips were imaged on the confocal microscopy (TCS SP8, Leica).

\section{STED superresolution for live cells}

Primary neuronal cultures were cultured at a suitable density (moderate) and seeded on glass-bottomed dishes. At DIV3, neurons were transfected with lentiviral vector to evaluate the gain-of-function of IncMtDloop. At DIV14, primary neuronal cultures were incubated with MitoESq-635 at a concentration of $1 \mu \mathrm{M}$ for 5 minutes in a humid atmosphere with $5 \%(\mathrm{v} / \mathrm{v}) \mathrm{CO}_{2}$ at $37^{\circ} \mathrm{C}$ to label the mitochondrial membrane, then imaged with STED nanoscopy. STED imaging was performed with Leica TCS SP8 STED $3 X$ system equipped with a white light laser for excitation and a 775-nm pulsed laser for STED depletion. A $\times 100$ oil-immersion objective (Leica, N.A. 1.4) was employed. 


\section{Electron microscopy}

The mice were perfused with paraformaldehyde-glutaraldehyde mixed fixative ( $2 \% / 2.5 \%)$, and the hippocampus was removed and post-fixed at $4^{\circ} \mathrm{C}$ using paraformaldehyde-glutaraldehyde mixed fixative $(2 \% / 2.5 \%)$ in $0.1 \mathrm{M} \mathrm{PB}(\mathrm{PH} 7.4)$. The hippocampal samples from each group were washed with $0.1 \mathrm{M} \mathrm{PB}$ (PH7.4) three times for $15 \mathrm{~min}$, and then post-fixed with $1 \%$ OsO4 for $2 \mathrm{~h}$ at $4{ }^{\circ} \mathrm{C}$, followed by serial ethanol dehydration and acetone transition for 5 min, embedding in Epon 812 resin, polymerization and polymerization at $60^{\circ} \mathrm{C}$ for $48 \mathrm{~h}$. The hippocampal samples were sliced into $60 \mathrm{~nm}$ for ultrathin sections of transmission electron microscopy with a Leica UC7 ultramicrotome. Ultrathin sections were loaded onto 100-mesh Cu grids and double stained with $2 \%$ uranyl acetate and lead citrate before observations employing a JEM 1400 plus transmission electron microscopy.

\section{RNA pulldown and mass spectrometry (MS)}

IncMtDloop was in vitro transcribed using the mMESSAGE mMACHINE T7 ULTRA Kit (Ambion, AMB1345), followed by biotinylation with the Pierce RNA 3' End Desthiobiotinylation Kit (Thermo Fisher Scientific, 20163) RNA pull-down was performed using a Magnetic RNA-protein Pull-Down Kit (Thermo Fisher Scientific, 20164) with the manufacturer's guidelines. The retrieved protein was detected by slivery staining (Thermo Fisher Scientific, 24612). The specific bands were identified by MS and western blot. For MS, specific bands and retrieved protein pull-downed by IncMtDloop or antisense probes were collected, and digested in $50 \mathrm{mM}$ triethylammonium bicarbonate with chymotrypsin and modified trypsin at $30^{\circ} \mathrm{C}$ overnight. After digestion, each peptide samples were extracted and speed vacuum-dried. Dried proteins were resuspended in $200 \mu$ l volume of buffer $(0.1 \%$ formic acid and $2 \%$ acetonitrile) and loaded on a Bruker Autoflex speed TOF/TOF MALDI-TOF system for LC/MS/MS. The acquired peptidesequencing data were loaded in Proteome Discoverer (version 1.3.0.339, Thermo Scientific) and analyzed with Mascot software (version 2.3.01, Matrix Science) against a Mus musculus protein database to identify proteins.

\section{RNA immunoprecipitation (RIP)}

RIP was performed using a Magnetic RIP RNA-Binding Protein Immunoprecipitation (Millipore, 17-700) in accordance with the manufacturer's guidelines. The primary cultured neurons were treated with ice-cold PBS and IP lysis buffer (Thermo Fisher Scientific, 87787) supplemented with RNase inhibitors and protease inhibitor cocktail. The supernatants were incubated with the anti-p32 (ab24733, Abcam) antibody or control mouse IgG with rotation at $4^{\circ} \mathrm{C}$ overnight, and then protein $\mathrm{A} / \mathrm{G}$ beads were added to each group. After the incubation at $4^{\circ} \mathrm{C}$ overnight, we collected the precipitates and extracted RNA by proteinase K-chloroform method. The enrichment of IncMtDloop was detected using qRT-PCR. 


\section{Luciferase reporter gene assays}

Luciferase assays were performed using the Dual-Luciferase ${ }^{\circledR}$ Reporter Assay Kit (Promega, E1960). N2a cells were planted in 96-well plates at $2.0 \times 10^{4}$ cells/well. After culturing for 2 days, si-RNA for TFAM was transfected into the cell lines by using Lipofectamine 2000 (Invitrogen, 11669019). After transfection for 2 days, cells were cotransfected with the luciferase reporter constructs (150ng/well) and internal control plasmid pRL-CMV (30ng/well). 2 days after transfection, the cells were collected to measure the luciferase activity using a luminometer according to the manufacturer's instructions.

\section{Chromatin immunoprecipitation (ChIP) assays}

N2A cells were collected at $\sim 90 \%$ confluency from $15 \mathrm{~cm}$ dishes, then performed CHIP assay using the SimpleChIP ${ }^{\circledR}$ Enzymatic Chromatin IP Kit (Cell Signaling, 9003). Firstly, cells were cross-linked with $37 \%$ formaldehyde for $10 \mathrm{~min}$, quenched, and digested with micrococcal nuclease for $20 \mathrm{~min}$ at $37^{\circ} \mathrm{C}$. The digested chromatin was sonicated in 500 $\mu$ l ChIP buffer on ice using a sonicator (three times, 20s each). Chromatin was Sheared chromatin was centrifugated at $9400 \mathrm{xg}$ for $10 \mathrm{~min}$ at $4^{\circ} \mathrm{C}$. The supernatant was collected and incubated with specific antibodies against TFAM (ab252432, Abcam) or IgG antibody at $4^{\circ} \mathrm{C}$ overnight with rotation, then incubated with protein $\mathrm{G}$ magnetic beads at $4^{\circ} \mathrm{C}$ for 2 hours with rotation. After purification, immunoprecipitated DNA was analysed by quantitative real-time PCR (40 cycles). Primer pairs must range from $150 \mathrm{bp}$ to $22 \mathrm{bp}$ long. The primer sequences of the promoter of mtDNA containing the TFAM binding domain were shown in the Supplementary Table 2.

\section{The IncRNA-protein interaction prediction}

The coding probability of IncMtDloop was examined with the PhyloCSF analysis in UCSC browser, Coding Potential Assessment Tool (CPAT, http://lilab.research.bcm.edu/cpat/index.php) and Coding Potential Calculator (CPC, http://cpc.cbi.pku.edu.cn/). The secondary structure of IncMtDloop was predicted on RNAfold WebServer (http://rna.tbi.univie.ac.at/cgi-bin/RNAfold.cgi) based on partition function and minimum free energy (MFE). The CatRAPID was used for the prediction of protein-RNA binding propensity between IncMtDloop fragments and possible proteins (http://service.tartaglialab.com/page/catrapid.group).

\section{Measure of extracellular fluxes using Seahorse XF96}

20,000 primary neurons were seeded in each well of a XF 96-well cell culture microplate in $80 \mathrm{ml}$ of culture media, and incubated overnight at $37^{\circ} \mathrm{C}$ in $5 \% \mathrm{CO}_{2}$. The four corners were left only with medium for background correction. Culture medium is replaced with $180 \mathrm{ml}$ of bicarbonate-free DMEM and cells 
are incubated at $37^{\circ} \mathrm{C}$ for 30 min before measurement. Oxygen consumption rates (OCR) were measured using a XF96 Extracellular Flux Analyzer ${ }^{74}$. OCR was determined at four levels: with no additions, and after adding: oligomycin $(1 \mu \mathrm{M})$; carbonyl cyanide 4-(trifluoromethoxy) phenylhydrazone (FCCP, $0.4 \mu \mathrm{M})$; and rotenone $(2 \mu \mathrm{M})$ (additives purchased from Sigma at highest quality). After each assay, manual inspection was performed on all wells using a conventionally light microscope. Wells for which the median OCR level did not follow the expected order, namely, median $\left(\mathrm{OCR}\left(\operatorname{Int}_{3}\right)\right)>$ median $\left(\mathrm{OCR}\left(\operatorname{Int}_{1}\right)\right)>$ median $\left(\mathrm{OCR}\left(\mathrm{Int}_{2}\right)\right)$ > median (OCR(Int 4$)$ ), were discarded (977 wells, 10.47\%). Of note, we excluded from the analysis contaminated wells and wells in which the cells got detached (461 wells, 4.94\%).

\section{A $B$ ELISA}

Hippocampus was isolated from 12-month-old 3×Tg mice. Hippocampal tissues were rinsed with PBS, and homogenized with a tissue homogenizer. The tissues were treated with an equal volume of RIPA buffer supplemented with protease inhibitor cocktail and lysed on ice for 30 minutes with gentle agitation. After centrifugation, aliquots of the lysates were removed and subjected to $A \beta$ measurement using $A \beta_{1-40}$ and $A \beta_{1-42}$ ELISA kits following the manufacturer's instructions.

\section{Cytochrome c Oxidase Assay}

Hippocampus was isolated from 12-month-old 3×Tg mice and subjected to Cytochrome c Oxidase measurement using Cytochrome $\mathrm{c}$ Oxidase Assay kits following the manufacturer's instructions. The colorimetric assay of the samples is based on observation of the decrease in absorbance at $550 \mathrm{~nm}$ of ferrocytochrome $\mathrm{c}$ caused by its oxidation to ferricytochrome $\mathrm{c}$ by cytochrome $\mathrm{c}$ oxidase.

\section{Electrophysiology}

LTP studies were performed on hippocampal slices from 12- month mice. Mice were anaesthetized with isoflurane, decapitated, and brains were rapidly removed in pre-ice cold ACSF $(120 \mathrm{mM} \mathrm{NaCl}, 2.5 \mathrm{mM} \mathrm{KCl}$, $1.25 \mathrm{mM} \mathrm{NaH}_{2} \mathrm{PO}_{4}, 26 \mathrm{mM} \mathrm{NaHCO}, 2 \mathrm{mM} \mathrm{MgSO}$, $10 \mathrm{mM}$ D-glucose, $2 \mathrm{mM} \mathrm{CaCl} 2$ with 95\% 02, 5\% CO2 [pH 7.4]). Transverse hippocampal slices were cut at a thickness of $350 \mu \mathrm{m}$, and placed on infusion chambers in ACSF. The fEPSPs were recorded from the CA1 stratum radiatum region by an extracellular borosilicate glass capillary pipette (resistances of 3-5 MQ) filled with ACSF. Stimulation of Schaffer collaterals from the CA3 region by a bipolar electrode. Signals were amplified using a MultiClamp $700 \mathrm{~B}$ amplifier (Axon), digitized using a Digidata 1440A Data Acquisition System (Axon) with $1 \mathrm{kHz}$ low pass filter and $2 \mathrm{kHz}$ high pass filter and analyzed using Clampex 10.7 software. For each experiment 8-9 sections from 3-4 animals/genotype were used. 


\section{Behavioral testing}

For nest building behavior, mice were individually house with nesting materials of facial tissue strips. Mice were given the nesting material on day 1 . Pictures were taken and nests were scored $24 \mathrm{~h}$ later. For open filed test, mice were placed into the open field arena (made of opaque white plastic material, $35 \mathrm{~cm}$ $\times 35 \mathrm{~cm}$ separated into $5 \times 5$ grids) by a blinded experimenter and allowed to explore the arena for $15 \mathrm{~min}$. The number of crossing grids and percentage of time spent in the center $(22 \mathrm{~cm} \times 22 \mathrm{~cm})$ were quantified using video tracking software. For novel object recognition test, the test was performed on three consecutive days. On the first day, mice were placed in the center of an empty open box and allowed to explore for $10 \mathrm{~min}$. On the second day, the mice were placed in the open box with two identical objects in the two corners and allowed to explore for $10 \mathrm{~min}$. On the third day, the mice were placed in the same box but with an object replaced by a novel one and allowed to explore for $10 \mathrm{~min}$. The discrimination index was referred to the time spent exploring the novel object relative to the time spent exploring both objects. For fear conditioning test, fear memory was measured by pairing a conditioned stimulus with an unconditioned stimulus. We used the Video Freeze Conditioning 'Video Freeze' software to record freezing behavior. On day one, mice were placed inside the chambers; after 120-s baseline, a 30-s CS tone followed, and a US foot shock was given during 120-150s, 180-210 s and 240-270 s. On day two, both contextual and cued phases were done. During phase one, mice were placed in the same testing chambers used on day one for $5 \mathrm{~min}$. During phase two, $3 \mathrm{~h}$ later, mice were placed into modified chambers with plastic inserts, and after $300 \mathrm{~s}$ the CS tine was played for $30 \mathrm{~s}$ at $1 \mathrm{~min}$ intervals (five CS tones in total). After $10 \mathrm{~min}$, mice were returned to their housing cages ${ }^{75,76}$. For morris water maze, the pool (150 cm diameter) filled with water that is maintained at room temperature. A $12 \mathrm{~cm}$ diameter transparent platform was placed $1 \mathrm{~cm}$ below the water surface at first quadrant. Mice were trained for 5 consecutive days, with 4 trials per day. Each training lasted $60 \mathrm{~s}$ until the mouse arrived on platform. After $24 \mathrm{~h}$ from the last training, the test was conducted without a platform for 60 s to test their memory performance. The latency time to the area of removed platform, the time spent in the first quadrant and number of crossing the area of removed platform was recorded.

\section{Quantification and Statistical analysis}

All statistical analysis was performed using GraphPad Prism 7 software. Results are showed as mean \pm SEM of three independent experiments. Significance between two groups was analyzed by two tailed Student's test. Significance between three groups or more was analyzed by one-way ANOVA or two-way ANOVA by Tukey's or Dunnett's multiple comparisons test. In all figures, ${ }^{*} \mathrm{P}<0.05,{ }^{* \star} \mathrm{P}<0.01,{ }^{* \star *} \mathrm{P}<0.001$, ${ }^{* \star \star \star} \mathrm{P}<0.0001, \mathrm{p}$ value of more than 0.05 was considered non-significant.

\section{Declarations}




\section{Acknowledgements}

We are grateful to Zhigang Yang from College of Physics and Optoelectronic Engineering, Shenzhen University for supplying the MitoESq-635 dye and thank Peng Xi and Zhaoyang Wu for the performance of STED. We also thank Yingqi Guo from Kunming Biological Diversity Regional Center for Instrument, Kunming Institute of Zoology, Chinese Academy of Science for her assistance in sample preparation and performing electron microscopy. This work was supported by grants from the National Natural Science Foundation of China (91649119 and 92049105), the Ministry of Science and Technology of China (2015CB755605), Peking University (BMU2019YJ001) and the key basic project of Yunnan province (E039030401) to J.L. This work was also supported by grants from the National Natural Science Foundation of China (NSFC 81821092) to L.L. The work was also supported, in part, by grants from the following: The Hong Kong Research Grants Council (RGC)-General Research Fund (GRF) (PI: GRF16100219 and GRF16100718); Alzheimer's Association Research Fellowship (PI: AARF-17-531566); Collaborative Research Fund (CRF) (Co-l: C4033-19EF); CUHK-Improvement on Competitiveness in Hiring New Faculties Funding Scheme (PI: Ref. 133) and CUHK-School of Life Sciences Startup funding to H.M.C.

\section{Author information}

These authors contributed equally: Wandi Xiong, Kaiyu Xu, Jacquelyne Ka-Li Sun.

Affiliations

Peking-Tsinghua Center for Life Sciences, Beijing, China

Wandi Xiong \& Lin Lu

\section{National Institute on Drug Dependence, Peking University, Beijing, China}

Wandi Xiong, Zhongyu Zhang, Fan Liao, Yongbo Zheng, Jie Shi, Lin Lu \& Jiali Li

Key Laboratory of Animal Models and Human Disease Mechanisms of Chinese Academy of Sciences \& Yunnan Province, Kunming Institute of Zoology, the Chinese Academy of Sciences, Kunming, Yunnan, China

Kaiyu Xu, Ying Zhang \& Jiali Li 
PKU/McGovern Institute for Brain Research, Peking University, Beijing, China

Wandi Xiong, Zhongyu Zhang, Fan Liao, Yongbo Zheng, Lin Lu \& Jiali Li

Kunming College of Life Science, University of Chinese Academy of Sciences,

Kunming, Yunnan, China

Kaiyu Xu \& Ying Zhang

School of Life Sciences, The Chinese University of Hong Kong, Hong Kong, China

Jacquelyne Ka-Li Sun \& Hei-Man Chow

Department of Neurobiology, The University of Pittsburgh School of Medicine, Pittsburgh, USA

Karl Herrup

Institute of Mental Health, National Clinical Research Center for Mental Disorders, Key Laboratory of Mental Health and Peking University Sixth Hospital, Peking University, Beijing, China

Wandi Xiong, Zhongyu Zhang, Fan Liao \& Lin Lu

National Resource Center for Non-Human Primates, Kunming Primate Research Center, and National Research Facility for Phenotypic and Genetic Analysis of Model Animals (Primate Facility), Kunming Institute of Zoology, Chinese Academy of Sciences, Kunming, China

Ying Zhang \& Jiali Li

Contributions

W.X. and J.L. conceived and designed all experiments and interpreted results. W. X., J.S., K.X., S.L., Y.Z., Z.Z., Z.W., L. F. and Zheng. Y. performed the experiments and analyzed the data. W.X. and J.L. designed 
and wrote the paper with assistance from P.X., H.C., H.K., S. J., and L.L. L.L. and J.L. supervised the project.

Corresponding authors

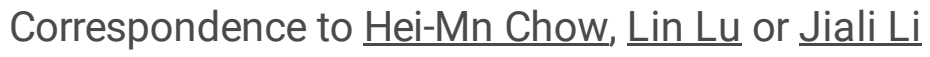

\section{Ethics declarations}

\section{Competing interests}

The authors declare no computing interests.

\section{References}

1. Sorrentino, V. Enhancing mitochondrial proteostasis reduces amyloid- $\beta$ proteotoxicity. Nature 552(2017).

2. Corbett, A., et al. Drug repositioning for Alzheimer's disease. Nat Rev Drug Discov 11, 833-846 (2012).

3. Perrin, R.J., Fagan, A.M. \& Holtzman, D.M. Multimodal techniques for diagnosis and prognosis of Alzheimer's disease. Nature 461, 916-922 (2009).

4. Lang, A.E. Clinical trials of disease-modifying therapies for neurodegenerative diseases: the challenges and the future. Nat Med 16, 1223-1226 (2010).

5. Mullard, A. Symptomatic AD treatment fails in first phase III. Nat Rev Drug Discov 15, 738 (2016).

6. Mullard, A. Failure of first anti-tau antibody in Alzheimer disease highlights risks of history repeating. Nat Rev Drug Discov 20, 3-5 (2021).

7. Fecher, C., et al. Cell-type-specific profiling of brain mitochondria reveals functional and molecular diversity. Nat Neurosci 22, 1731-1742 (2019).

8. Reddy, P.H., et al. Amyloid-beta and mitochondria in aging and Alzheimer's disease: implications for synaptic damage and cognitive decline. J Alzheimers Dis 20 Suppl 2, S499-512 (2010).

9. Balaban, R.S., Nemoto, S. \& Finkel, T. Mitochondria, oxidants, and aging. Cel/ 120, 483-495 (2005).

10. Linnane, A.W., Marzuki, S., Ozawa, T. \& Tanaka, M. Mitochondrial DNA mutations as an important contributor to ageing and degenerative diseases. Lancet 1, 642-645 (1989). 
11. Wallace, D.C. Mitochondrial genetics: a paradigm for aging and degenerative diseases? Science 256, 628-632 (1992).

12. Hirai, K., et al. Mitochondrial abnormalities in Alzheimer's disease. J Neurosci 21, 3017-3023 (2001).

13. Kujoth, G.C., et al. Mitochondrial DNA mutations, oxidative stress, and apoptosis in mammalian aging. Science 309, 481-484 (2005).

14. Lane, N. \& Martin, W. The energetics of genome complexity. Nature 467, 929-934 (2010).

15. Cai, N., et al. Mitochondrial DNA variants modulate $\mathrm{N}$-formylmethionine, proteostasis and risk of late-onset human diseases. Nat Med 27, 1564-1575 (2021).

16. Sabouny, R. \& Shutt, T.E. The role of mitochondrial dynamics in mtDNA maintenance. J Cell Sci 134(2021).

17. Du, H., et al. Early deficits in synaptic mitochondria in an Alzheimer's disease mouse model. Proc Natl Acad Sci U S A 107, 18670-18675 (2010).

18. Sorrentino, V., et al. Enhancing mitochondrial proteostasis reduces amyloid-beta proteotoxicity. Nature 552, 187-193 (2017).

19. Rinn, J.L. \& Chang, H.Y. Genome regulation by long noncoding RNAs. Annu Rev Biochem 81, 145166 (2012).

20. Pollard, K.S., et al. An RNA gene expressed during cortical development evolved rapidly in humans. Nature 443, 167-172 (2006).

21. Qureshi, I.A. \& Mehler, M.F. Emerging roles of non-coding RNAs in brain evolution, development, plasticity and disease. Nat Rev Neurosci 13, 528-541 (2012).

22. Liu, S., et al. Annotation and cluster analysis of spatiotemporal- and sex-related IncRNA expression in rhesus macaque brain. Genome Res 27, 1608-1620 (2017).

23. Vance, K.W. \& Ponting, C.P. Transcriptional regulatory functions of nuclear long noncoding RNAs. Trends Genet 30, 348-355 (2014).

24. Wang, K.C. \& Chang, H.Y. Molecular mechanisms of long noncoding RNAs. Mol Cell 43, 904-914 (2011).

25. Geisler, S. \& Coller, J. RNA in unexpected places: long non-coding RNA functions in diverse cellular contexts. Nat Rev Mol Cell Biol 14, 699-712 (2013). 
26. Palazzo, A.F. \& Koonin, E.V. Functional Long Non-coding RNAs Evolve from Junk Transcripts. Cell $183,1151-1161$ (2020).

27. Salta, E. \& De Strooper, B. Non-coding RNAs with essential roles in neurodegenerative disorders. Lancet Neurol 11, 189-200 (2012).

28. Statello, L., Guo, C.J., Chen, L.L. \& Huarte, M. Gene regulation by long non-coding RNAs and its biological functions. Nat Rev Mol Cell Biol 22, 96-118 (2021).

29. Rackham, O., et al. Long noncoding RNAs are generated from the mitochondrial genome and regulated by nuclear-encoded proteins. RNA 17, 2085-2093 (2011).

30. Mercer, T.R., et al. The human mitochondrial transcriptome. Cell 146, 645-658 (2011).

31. Gao, Y., et al. Long non-coding RNA ASncmtRNA-2 is upregulated in diabetic kidneys and high glucose-treated mesangial cells. Exp Ther Med 13, 581-587 (2017).

32. Villegas, J., et al. Expression of a novel non-coding mitochondrial RNA in human proliferating cells. Nucleic Acids Res 35, 7336-7347 (2007).

33. Dorn, G.W., 2nd. LIPCAR: a mitochondrial Inc in the noncoding RNA chain? Circ Res 114, 15481550 (2014).

34. Kumarswamy, R., et al. Circulating long noncoding RNA, LIPCAR, predicts survival in patients with heart failure. Circ Res 114, 1569-1575 (2014).

35. Hillen, H. The Beta Amyloid Dysfunction (BAD) Hypothesis for Alzheimer's Disease. Front Neurosci 13, 1154 (2019).

36. Ferre, F., Colantoni, A. \& Helmer-Citterich, M. Revealing protein-IncRNA interaction. Brief Bioinform 17, 106-116 (2016).

37. Agostini, F., et al. catRAPID omics: a web server for large-scale prediction of protein-RNA interactions. Bioinformatics 29, 2928-2930 (2013).

38. Bellucci, M., Agostini, F., Masin, M. \& Tartaglia, G.G. Predicting protein associations with long noncoding RNAs. Nat Methods 8, 444-445 (2011).

39. Livi, C.M., Klus, P., Delli Ponti, R. \& Tartaglia, G.G. catRAPID signature: identification of ribonucleoproteins and RNA-binding regions. Bioinformatics 32, 773-775 (2016).

40. Barna, J., et al. Complement component 1q subcomponent binding protein in the brain of the rat. Sci Rep 9, 4597 (2019). 
41. Gotoh, K., et al. Mitochondrial p32/C1qbp Is a Critical Regulator of Dendritic Cell Metabolism and Maturation. Cell Rep 25, 1800-1815 e1804 (2018).

42. Jiang, J., Zhang, Y., Krainer, A.R. \& Xu, R.M. Crystal structure of human p32, a doughnut-shaped acidic mitochondrial matrix protein. Proc Natl Acad Sci U S A 96, 3572-3577 (1999).

43. Leucci, E., et al. Melanoma addiction to the long non-coding RNA SAMMSON. Nature 531, 518522 (2016).

44. Yagi, M., et al. p32/gC1qR is indispensable for fetal development and mitochondrial translation: importance of its RNA-binding ability. Nucleic Acids Res 40, 9717-9737 (2012).

45. Baloyannis, S.J. Mitochondrial alterations in Alzheimer's disease. J Alzheimers Dis 9, 119-126 (2006).

46. Wang, W., Zhao, F., Ma, X., Perry, G. \& Zhu, X. Mitochondria dysfunction in the pathogenesis of Alzheimer's disease: recent advances. Mol Neurodegener 15, 30 (2020).

47. Yu, H., et al. Mitochondrial Molecular Abnormalities Revealed by Proteomic Analysis of Hippocampal Organelles of Mice Triple Transgenic for Alzheimer Disease. Front Mol Neurosci 11, 74 (2018).

48. Muta, T., Kang, D., Kitajima, S., Fujiwara, T. \& Hamasaki, N. p32 protein, a splicing factor 2associated protein, is localized in mitochondrial matrix and is functionally important in maintaining oxidative phosphorylation. J Biol Chem 272, 24363-24370 (1997).

49. Zhunina, O.A., et al. Neurodegenerative Diseases Associated with Mitochondrial DNA Mutations. Curr Pharm Des 26, 103-109 (2020).

50. Long, Q., et al. Phase separation drives the self-assembly of mitochondrial nucleoids for transcriptional modulation. Nat Struct Mol Bio/ 28, 900-908 (2021).

51. Choi, W.S. \& Garcia-Diaz, M. A minimal motif for sequence recognition by mitochondrial transcription factor A (TFAM). Nucleic Acids Res 50, 322-332 (2022).

52. Bruser, C., Keller-Findeisen, J. \& Jakobs, S. The TFAM-to-mtDNA ratio defines inner-cellular nucleoid populations with distinct activity levels. Cell Rep 37, 110000 (2021).

53. Hillen, H.S., Morozov, Y.I., Sarfallah, A., Temiakov, D. \& Cramer, P. Structural Basis of Mitochondrial Transcription Initiation. Cell 171, 1072-1081 e1010 (2017).

54. Hillen, H.S., Temiakov, D. \& Cramer, P. Structural basis of mitochondrial transcription. Nat Struct Mol Biol 25, 754-765 (2018). 
55. Karbowski, M. \& Youle, R.J. Dynamics of mitochondrial morphology in healthy cells and during apoptosis. Cell Death Differ 10, 870-880 (2003).

56. Vicidomini, G., Bianchini, P. \& Diaspro, A. STED super-resolved microscopy. Nat Methods 15, 173182 (2018).

57. Yang, X., et al. Mitochondrial dynamics quantitatively revealed by STED nanoscopy with an enhanced squaraine variant probe. Nat Commun 11, 3699 (2020).

58. Liesa, M. \& Shirihai, O.S. Mitochondrial dynamics in the regulation of nutrient utilization and energy expenditure. Cell Metab 17, 491-506 (2013).

59. Wilson, D.F., Harrison, D.K. \& Vinogradov, A. Mitochondrial cytochrome c oxidase and control of energy metabolism: measurements in suspensions of isolated mitochondria. J Appl Physiol (1985) 117, 1424-1430 (2014).

60. Kerr, J.S., et al. Mitophagy and Alzheimer's Disease: Cellular and Molecular Mechanisms. Trends Neurosci 40, 151-166 (2017).

61. Kingwell, K. Turning up mitophagy in Alzheimer disease. Nat Rev Drug Discov (2019).

62. Spillantini, M.G. \& Goedert, M. Tau pathology and neurodegeneration. Lancet Neuro/ 12, 609-622 (2013).

63. Fang, E.F., et al. Mitophagy inhibits amyloid-beta and tau pathology and reverses cognitive deficits in models of Alzheimer's disease. Nat Neurosci 22, 401-412 (2019).

64. Kapogiannis, D. \& Mattson, M.P. Disrupted energy metabolism and neuronal circuit dysfunction in cognitive impairment and Alzheimer's disease. Lancet Neuro/ 10, 187-198 (2011).

65. Khacho, M., et al. Mitochondrial dysfunction underlies cognitive defects as a result of neural stem cell depletion and impaired neurogenesis. Hum Mol Genet 26, 3327-3341 (2017).

66. Faghihi, M.A., et al. Expression of a noncoding RNA is elevated in Alzheimer's disease and drives rapid feed-forward regulation of beta-secretase. Nat Med 14, 723-730 (2008).

67. Mus, E., Hof, P.R. \& Tiedge, H. Dendritic BC200 RNA in aging and in Alzheimer's disease. Proc Natl Acad Sci U S A 104, 10679-10684 (2007).

68. Rogaeva, E., et al. The neuronal sortilin-related receptor SORL1 is genetically associated with Alzheimer disease. Nat Genet 39, 168-177 (2007).

69. Litvinchuk, A., et al. Complement C3aR Inactivation Attenuates Tau Pathology and Reverses an Immune Network Deregulated in Tauopathy Models and Alzheimer's Disease. Neuron 100, 1337-1353 e1335 (2018). 
70. Lee, C.Y.D., et al. Elevated TREM2 Gene Dosage Reprograms Microglia Responsivity and Ameliorates Pathological Phenotypes in Alzheimer's Disease Models. Neuron 97, 1032-1048 e1035 (2018).

71. Wyse, A.T., Siebert, C., Bobermin, L.D., Dos Santos, T.M. \& Quincozes-Santos, A. Changes in Inflammatory Response, Redox Status and $\mathrm{Na}(+), \mathrm{K}(+)$-ATPase Activity in Primary Astrocyte Cultures from Female Wistar Rats Subject to Ovariectomy. Neurotox Res 37, 445-454 (2020).

72. Mclnnes, J., et al. Synaptogyrin-3 Mediates Presynaptic Dysfunction Induced by Tau. Neuron 97, 823-835 e828 (2018).

73. Ising, C., et al. NLRP3 inflammasome activation drives tau pathology. Nature 575, 669-673 (2019).

74. Yepez, V.A., et al. OCR-Stats: Robust estimation and statistical testing of mitochondrial respiration activities using Seahorse XF Analyzer. PLoS One 13, e0199938 (2018).

75. laccarino, H.F., et al. Gamma frequency entrainment attenuates amyloid load and modifies microglia. Nature 540, 230-235 (2016).

76. Hou, Y. NAD+ supplementation normalizes key Alzheimer's features and DNA damage responses in a new AD mouse model with introduced DNA repair deficiency. Proc. Natl Acad. Sci. USA 115(2018).

\section{Figures}



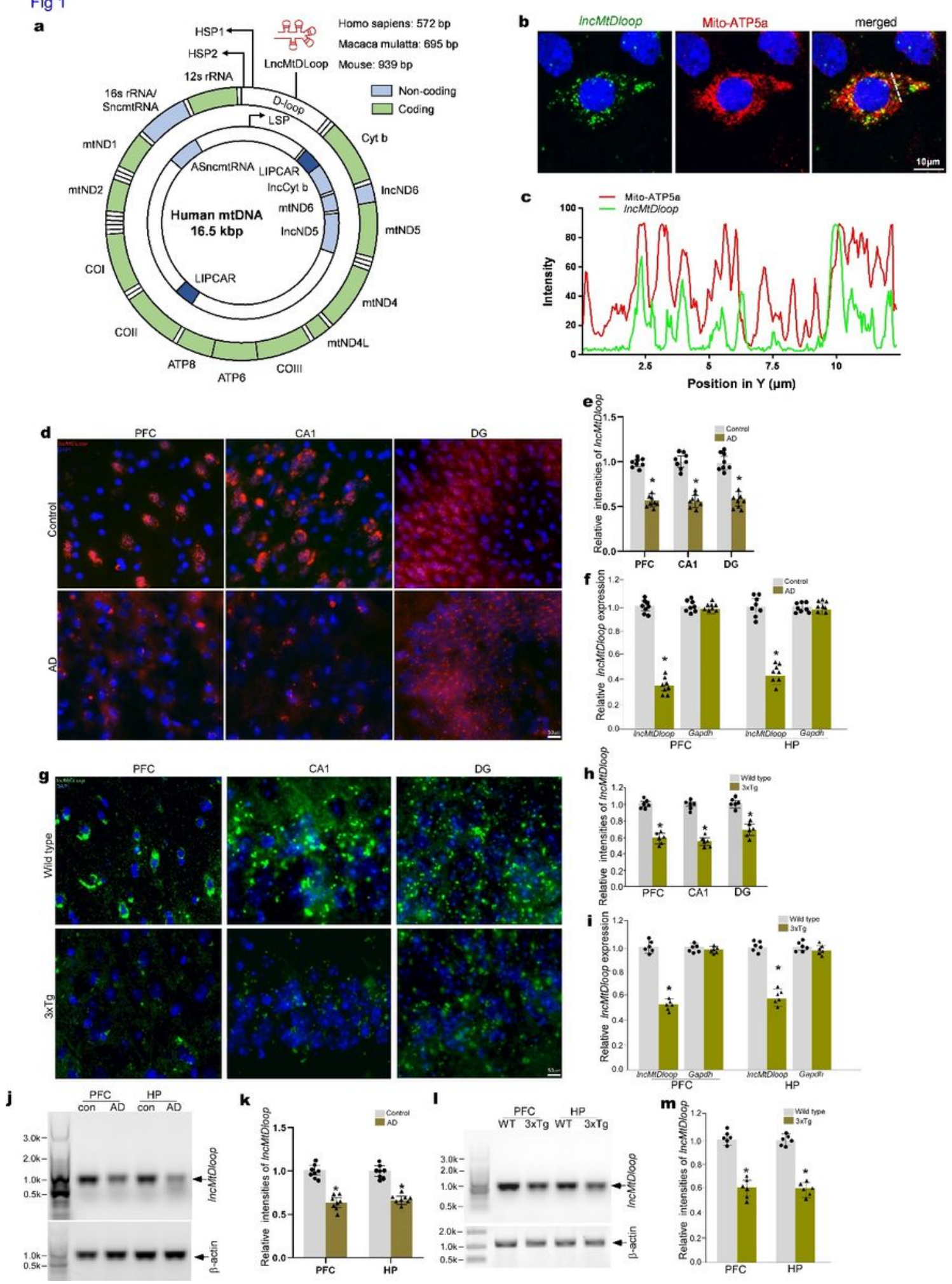

\section{Figure 1}

Decrease in IncMtDloop expression found in the brains of human AD patients and the mouse model. (a) Schematic illustrating the detailed structure of mitochondrial non-coding region, D-loop, and transcripts of IncMtDloop in human, macaque and mouse. The two strands of mtDNA are indicated as the heavy and light strands. Abbreviations: CBS, conserved sequence block; D-loop, displacement loop; HSP, heavy strand promoter; HVS, hypervariable segment; LSP, light strand promoter; TAS, termination associated 
sequence. (b) Representative images of RNAscope ISH together with immunofluorescence showing the location of IncMtDloop and mitochondria in hippocampal neurons of wild type mouse. (c) Relative intensities of IncMtDloop and mito-ATP5a fluorescence signals illustrated in white dotted line in b) were quantified by Image J. (d) Representative images of RNAscope ISH showing IncMtDloop expression. 15 $\mu \mathrm{m}$ paraffin sections of prefrontal cortex (PFC) and hippocampus (CA1 and DG) from age- and sexmatched control and $A D$ individuals were performed RNAscope with human- specific probe sets against IncMtDloop. Scale bar, $10 \mu \mathrm{m}$. (e) Relative intensity of RNAscope fluorescence illustrated in A) were quantified by Image J. Bars indicate mean \pm SEM. $n=8$ patients per group. ${ }^{*} \mathrm{P}<0.05$, by unpaired $t$ - test. (f) SsRT-qPCR was performed for validation of IncMtDloop expression. Total RNAs were extracted from postmortem frozen PFC and hippocampal tissues of age- and sex-matched control and AD individuals. Bars indicate mean \pm SEM. $n=8$ patients per group. ${ }^{*} P<0.05$, by unpaired $t$-test. (g) Representative images of RNAscope ISH showing IncMtDloop expression. $10 \mu \mathrm{m}$ cryostat brain sections from 12-monthold wild type and 3xTg mice were performed RNAscope with mouse-specific probe sets against IncMtDloop. Scale bar, $15 \mu \mathrm{m}$. (h) Relative intensity of RNAscope ISH fluorescence illustrated in D) are quantified by Image J. Bars indicate mean \pm SEM. $n=6$ mice per genotype. ${ }^{*} P<0.05$, by unpaired $t$-test. (i) ssRT-qPCR was performed for validation of IncMtDLoop expression. Total RNAs were extracted from fresh PFC and hippocampal tissues of 12-month-old wild type and 3xTg mice. Bars indicate mean \pm SEM. $\mathrm{n}=6$ mice per group. ${ }^{*} \mathrm{P}<0.05$, by unpaired t-test. (j) Representative northern blots of IncMtDloop transcript in postmortem frozen PFC and hippocampal tissues of control and AD individuals. (k)Relative intensities of northern blot signals illustrated in panel i) were quantified by use of Image J. Bars indicate mean \pm SEM. $n=8$. ${ }^{*} \mathrm{P}<0.05$, by unpaired t-test. (I) Representative northern blots of IncMtDloop transcript in fresh PFC and hippocampal tissues of wild type and $3 x T g$ mice. Bars indicate mean \pm SEM. $n=6$. *P $<0.05$, by unpaired t-test. $(\mathrm{m})$ Relative intensities of northern blot signals illustrated in panel $\mathrm{I}$ ) were quantified by use of Image $J$. Bars indicate mean \pm SEM. $n=6$ mice per genotype. ${ }^{*} P<0.05$, by unpaired $t-$ test. 
Fig 2

a

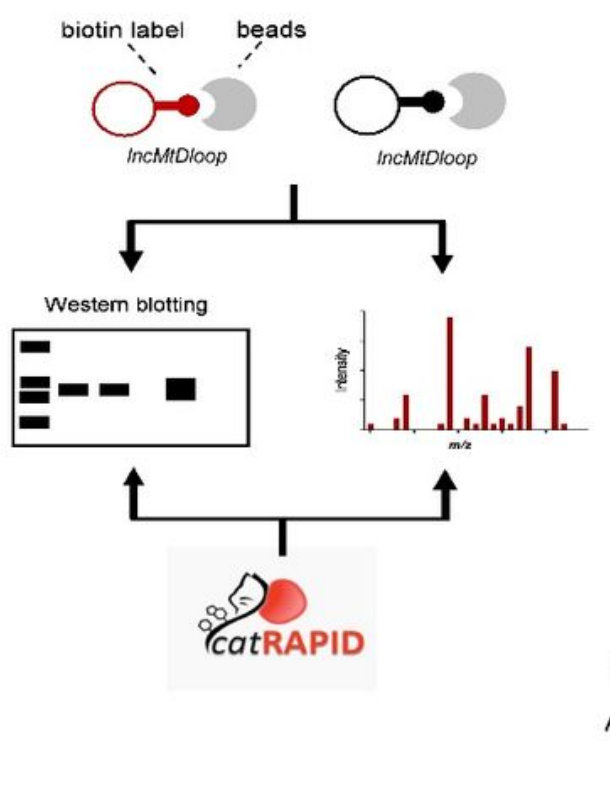

b

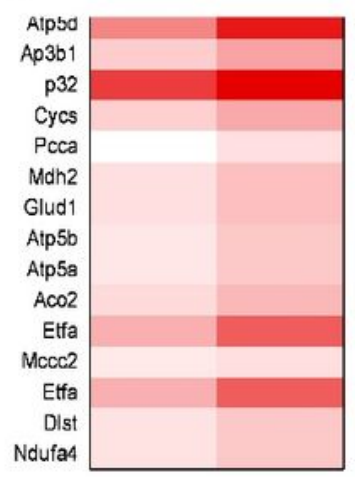

c

50

0

$-50$

$-100$
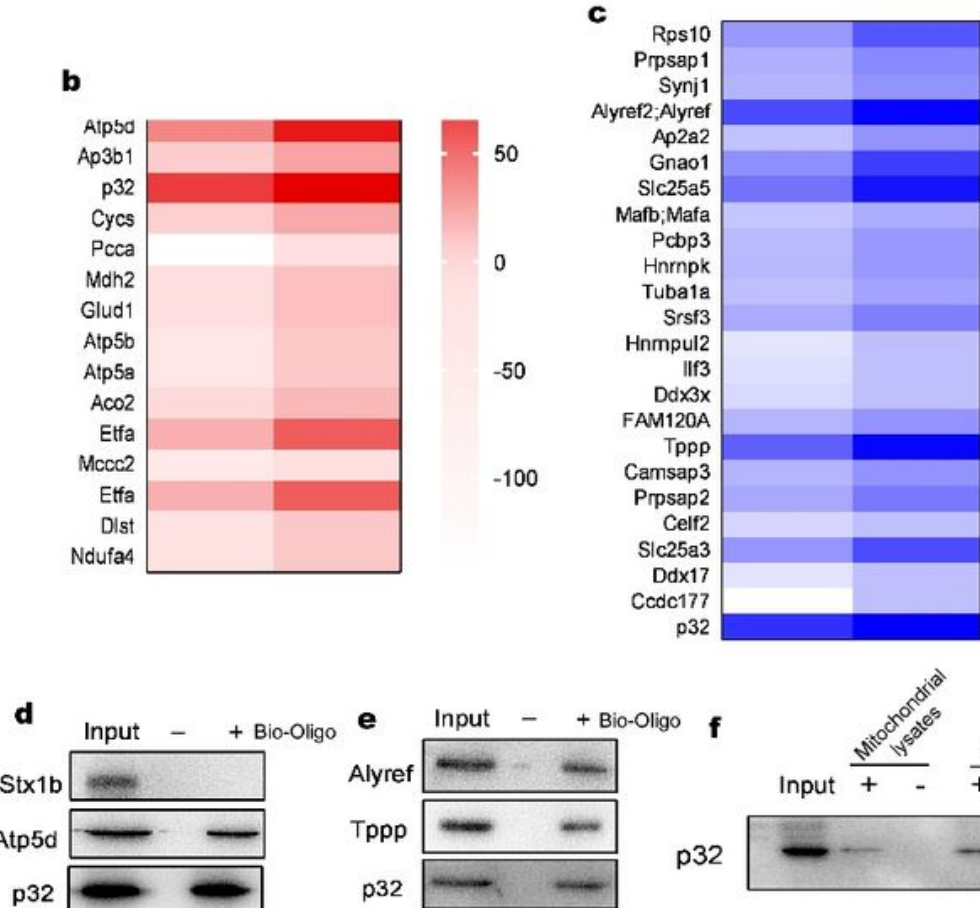

p32
100

$\mathbf{g}$

RNA-protein interaction(IncMtDloop)

Interaction propensity $=71$ Discriminative power $=98 \%$

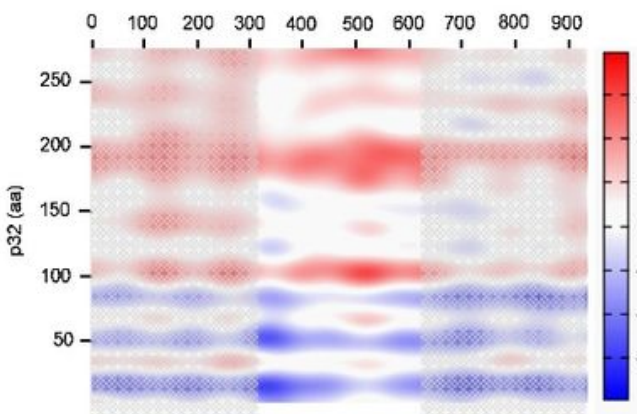

h
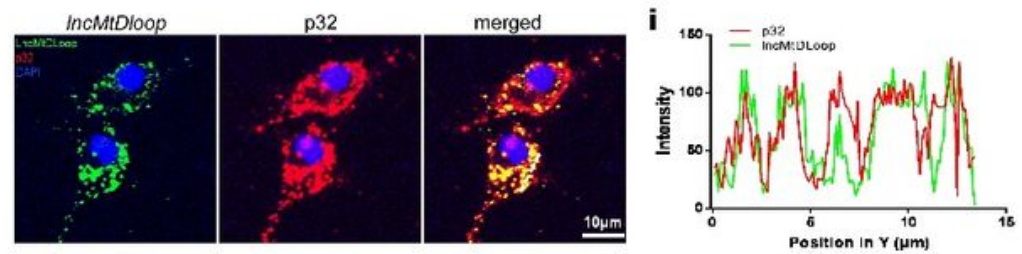

j
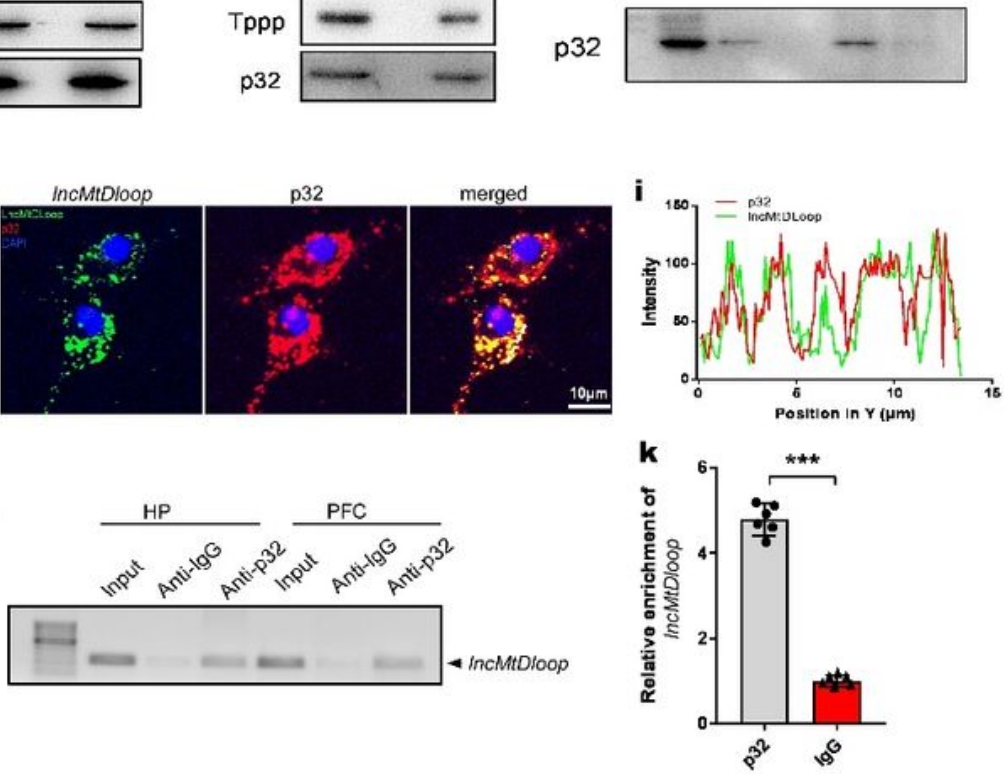

I

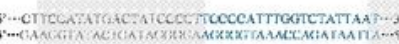

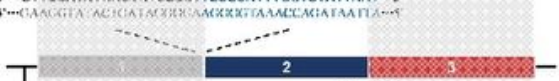

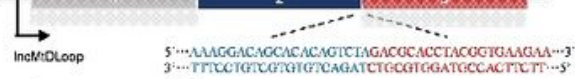
313
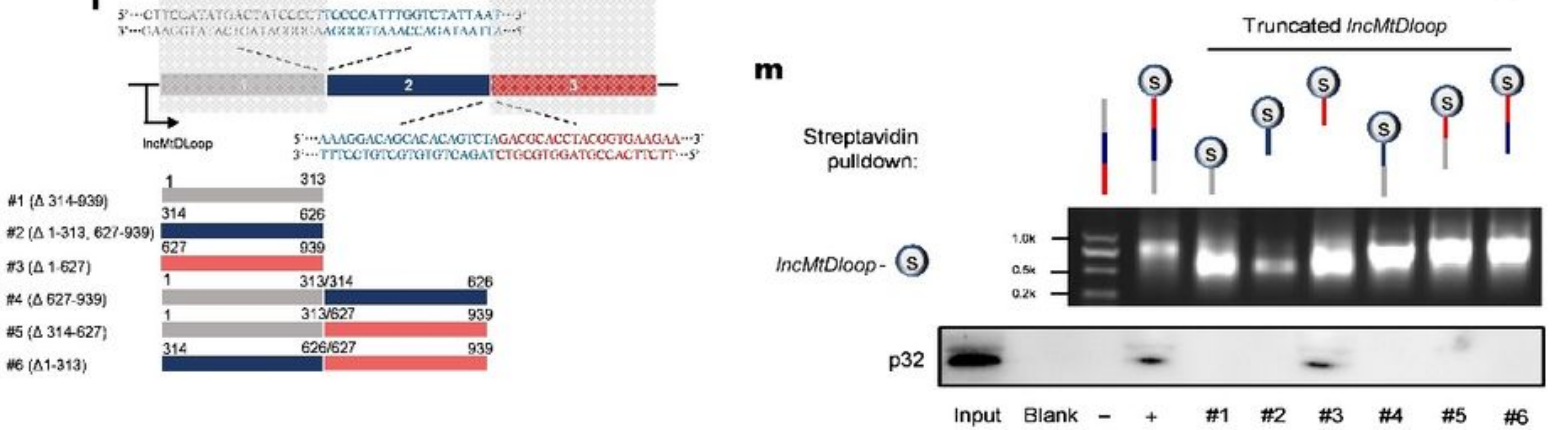

Figure 2

LncMtDloop binds to mitochondria-related proteins. (a) Schematic illustrating the procedure of IncMtDloop RNA pull-down and mass spectrum (MS). (b) The interaction propensity of IncMtDloop with binding partners in mitochondrial preparations from hippocampal tissues of 6-month-old wild type mice, predicted by CatRAPID, referring the results of mass spectrum. The left row indicates the scores of interaction propensity of the proteins. The right row indicates the discriminative power of the proteins, 
representing as centesimal system. (c) The interaction propensity of IncMtDloop with binding partners in cytosolic preparations from hippocampal tissues of 6-month-old wild type mice, predicted by CatRAPID, referring the results of mass spectrum. The left row indicates the scores of interaction propensity of the proteins. The right row indicates the discriminative power of the proteins, representing as centesimal system. (d) RNA pull-down using biotinylated IncMtDloop and antisense-IncMtDloop in mitochondrial preparations of hippocampal tissues of 6-month-old wildtype mice, following by western blot of Stx $1 \mathrm{~b}$, ATP5d and p32. +, IncMtDloop; - , anti-IncMtDloop. (e) RNA pull-down using biotinylated IncMtDloop and antisense-IncMtDloop in cytosolic preparations of hippocampal tissues of 6-month-old wildtype mice, following by western blot of Stx1b, ATP5d and p32. +, IncMtDLoop; -, anti-IncMtDloop. (f) RNA pull-down using biotinylated IncMtDloop and antisense-IncMtDLoop in mitochondrial and cytosolic preparations, respectively, from hippocampal tissues of 6-month-old wildtype mice, following by western blot of p32. +, IncMtDloop; - , anti-IncMtDloop. (g) Heatmap illustrating the interaction propensity of the IncMtDloop binding to $\mathrm{p} 32$, as predicted by CatRAPID. The $X$ axis represents RNA nucleotide sequence location, and the $Y$ axis represents the protein residue location. Colors scale bar with red shades indicate the predictions with high interaction propensity. (h) Representative images of RNAscope ISH and immunostaining showing co- localization of IncMtDloop (green) and p32 (red) in wild type hippocampal neurons. (i) Relative intensities of fluorescence signals of IncMtDloop and p32 illustrated in panel H) were quantified by use of Image J. (j) Representative northern blots of IncMtDLoop transcript in the RIP assay by p32 in extract preparations from fresh brain tissues of 12-old-month wild type mice. (k) qPCR for detectable IncMtDloop from RIP analysis of p32 in preparations of fresh hippocampal tissues of 12month-old wild type mice. Results were normalized to the control IgG RIP group. Bars indicate mean \pm SEM. $\mathrm{n}=6$ animals per group. ${ }^{* \star} \mathrm{P}<0.001$, by unpaired $\mathrm{t}$-test. (I) Schematic map for constructing six truncated mutants of IncMtDloop to identify its protein-binding motifs. (m) RNA pull-down using biotinylated distinct fragments of IncMtDloop and antisense-IncMtDloop in mitochondrial preparations from hippocampal tissues as described above, following by western blot of $\mathrm{p} 32$. The indicated six truncates of IncMtDloop were transcribed in vitro and tagged-RNA streptavidin to perform RNA pull-down assays. 
Fig 3
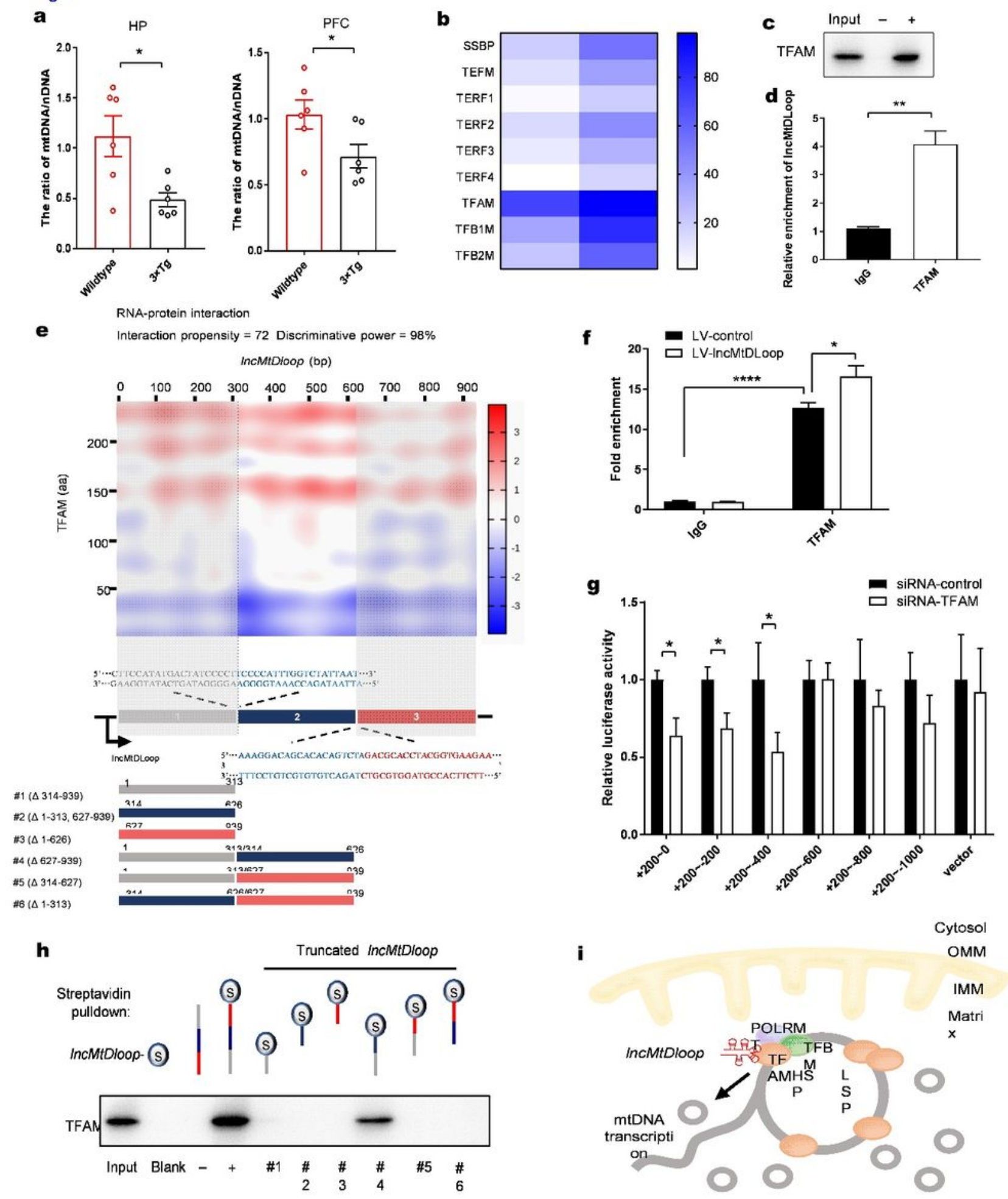

i

Cytosol ОМM

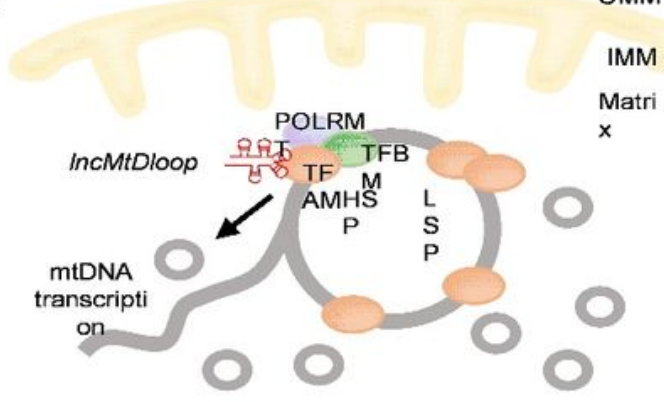

Figure 3

LncMtDloop recruits TFAM to promote the mitochondrial transcription initiation. (a) qPCR was performed for validation of ratio of mtDNA/nDNA expression. Total RNAs were extracted from fresh hippocampal and PFC tissues of 12-month-old wild type and 3xTg mice. Bars indicate mean \pm SEM. $n=6$ mice per group. ${ }^{*} \mathrm{P}<0.05$, by unpaired t-test. (b) The interaction propensity of IncMtDloop with potential binding proteins in mitochondrial nucleoid predicted by CatRAPID, The left row indicates the scores of interaction 
propensity of the proteins. The right row indicates the discriminative power of the proteins, representing as centesimal system. SSBP, mitochondrial single stranded DNA-binding protein; TEFM, mitochondrial elongator factor; TERF1-4, mitochondrial transcription termination factors 1-4; TFAM, mitochondrial transcription factor A; TFB1M/TFB2M, mitochondrial transcription factors B1/B2. (c) RNA pull-down using biotinylated IncMtDloop and antisense-IncMtDLoop in mitochondrial preparations from hippocampal tissues of 6-month-old wildtype mice, following by western blot of TFAM. +, IncMtDloop; -, anti-IncMtDloop. (d) qPCR for detectable IncMtDloop from RIP analysis of TFAM in preparations of fresh hippocampal tissues of 12-month-old wild type mice. Results were normalized to the control IgG RIP group. Bars indicate mean \pm SEM. $n=6$ animals per group. ${ }^{\star \star} P<0.01$, by unpaired t-test. (e) Heatmap illustrating the interaction propensity of the IncMtDloop binding to TFAM, as predicted by CatRAPID. The $X$ axis represents RNA nucleotide sequence location, and the $Y$ axis represents the protein residue location. Colors scale bar with red shades indicate the predictions with high interaction propensity. $(\mathrm{f})$ Mitochondrial promoter within D-loop region is sufficient for the biding of TFAM by ChIP-RNA analysis. Bars indicate mean \pm SEM. $n=3$ per group. ${ }^{*} P<0.05$, ${ }^{\star} \star \star * P<0.0001$, by unpaired $t-$ test. (g) Six dieffrent loci of mitochondrial promoter in D-loop regions were constructed into pGL4.10 vector and performed luciferase reporter assays in TFAM-silenced N2a cells. Bars indicate mean \pm SEM. $n=8-10$ per group. *P $<0.05$, by unpaired t- test. (i) Schematic illustrating the mechanism of IncMtDloop and TFAM. 
Fig 4

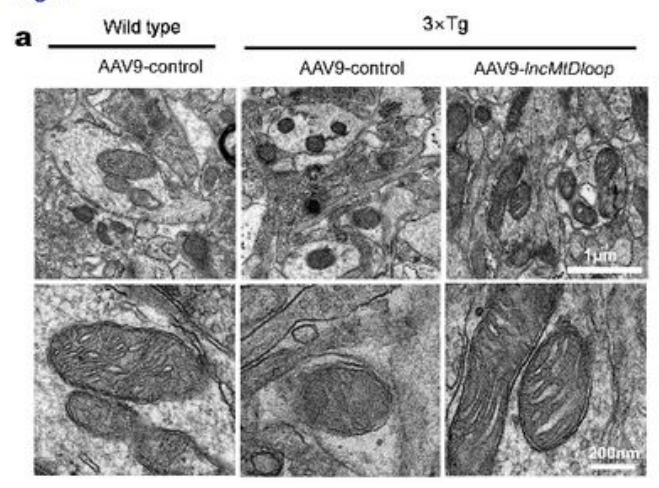

b $\square$ Tubular $\square$ Short tubular $\boldsymbol{E}$ Fragmented $\square$ Large spherical
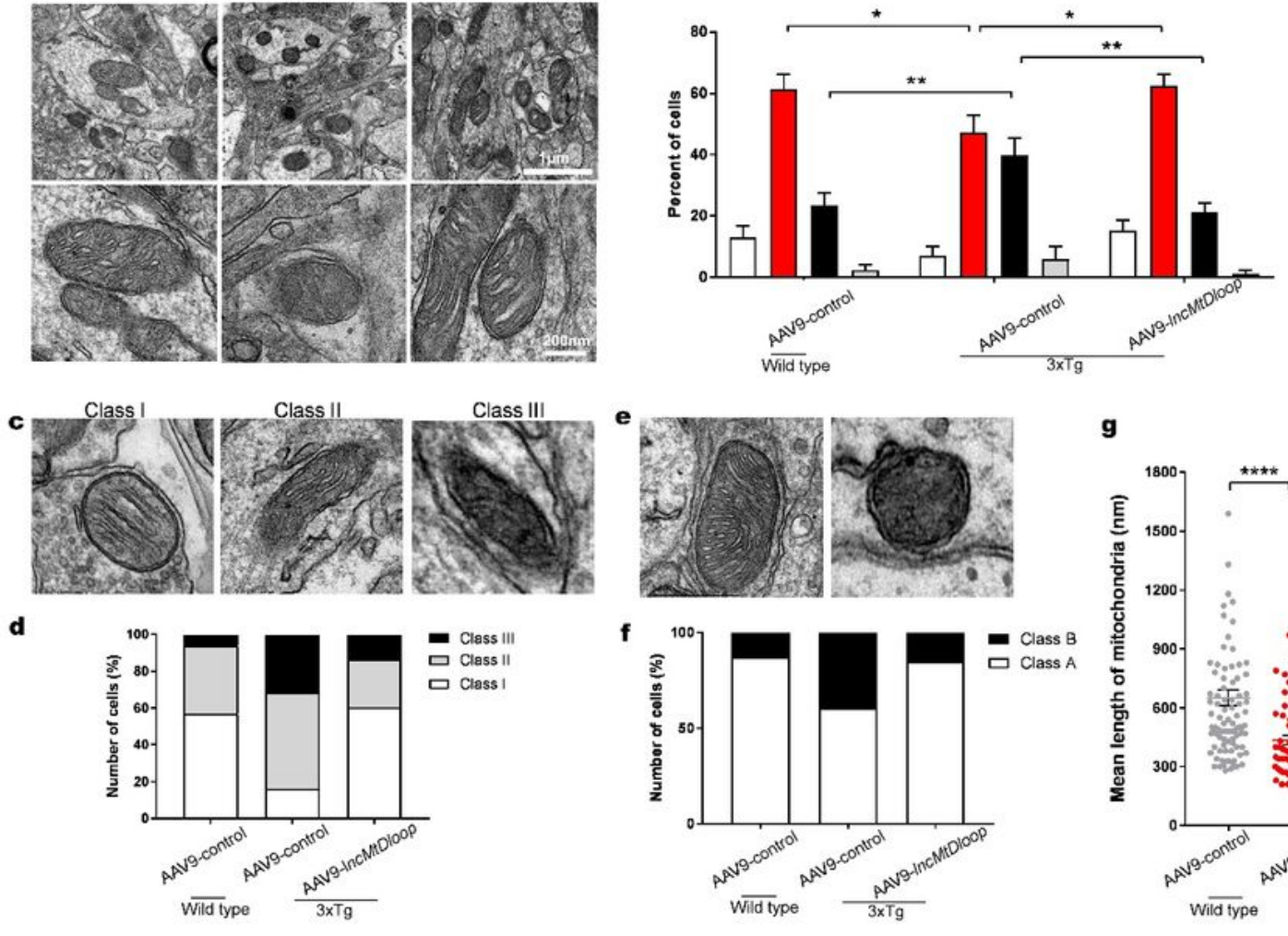

d
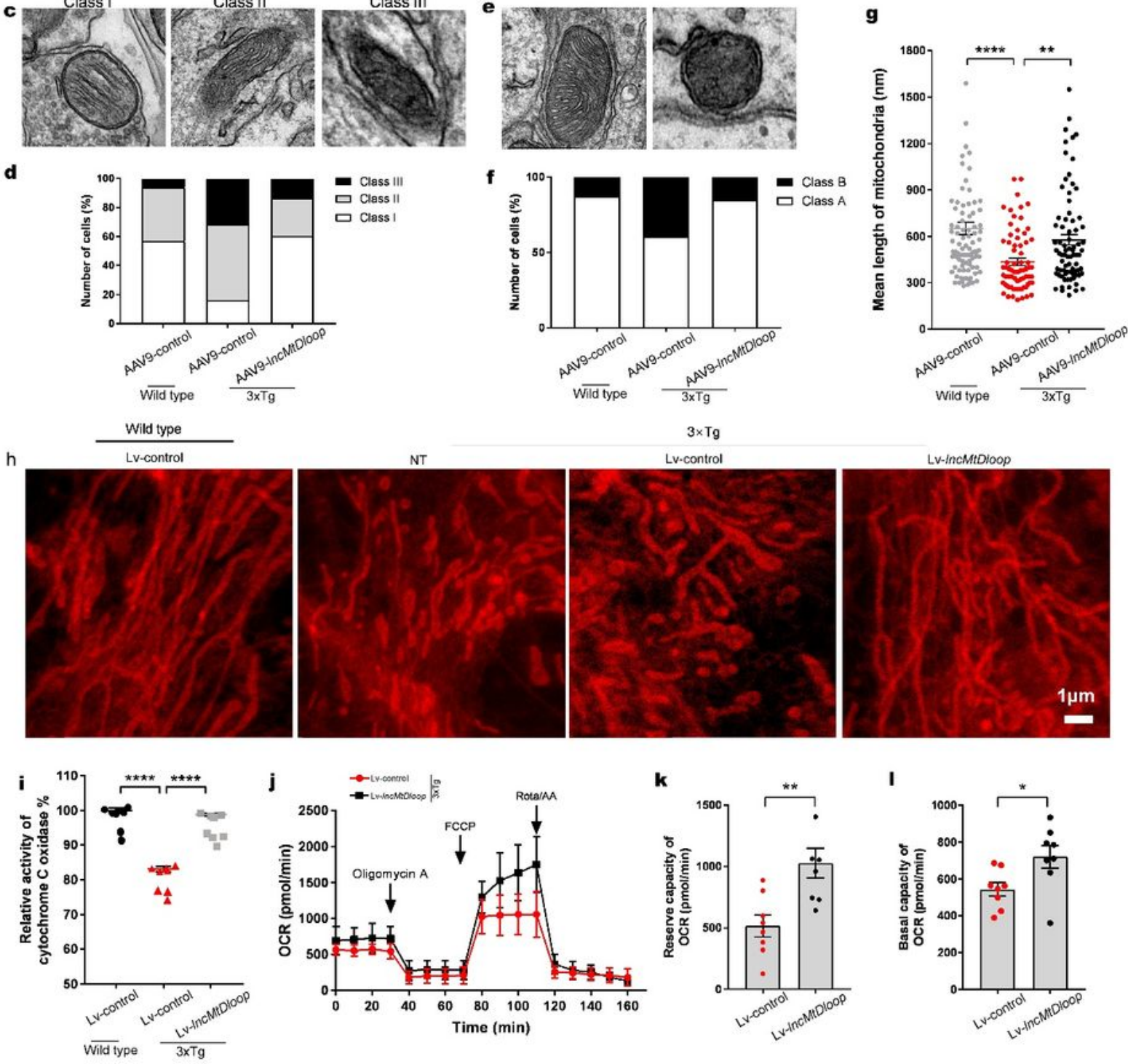

Figure 4

Restoring IncMtDloop expression alleviates AD-related alterations in mitochondrial morphology and function. (a) Representative electron micrographs showing mitochondrial ultrastructure of hippocampal neurons of 12-month-old wild type and 3xTg mice w/o overexpressing IncMtDloop. $\mathrm{n}=10$ random fields from hippocampal tissue samples of 6 animals per group. (b) Percentage of changes in mitochondrial morphology (tubular, short tubular, fragmented and large spherical) was quantified in hippocampal 
neurons as described above. Bars indicate mean \pm SEM. $\mathrm{n}=6$ per group. ${ }^{\star} P<0.05,{ }^{\star} * P<0.01$, by one-way ANOVA with Tukey's multiple comparisons test. ( $c$ and d) Representative electron micrographs of mitochondrial cristae number (top), and percentage of cells with different categories: Class I (more than three cristae), Class II (two to three cristae) and Class III (no or one cristae), in hippocampal neurons as

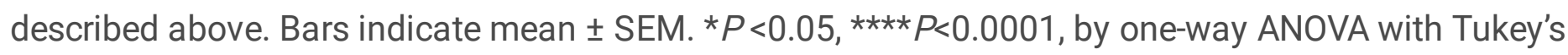
multiple comparisons test. ( $d$ and $f$ ) Representative electron micrographs of mitochondrial matrix density (bottom), and percentage of cells with different categories: Class A (dense matrix), Class B (swollen mitochondria with hypodense matrix), in hippocampal neurons as described above. Bars indicate mean \pm SEM. ${ }^{* \star \star} P<0.001, * \star \star \star P<0.0001$, by one-way ANOVA with Tukey's multiple comparisons test. (g) Quantification of changes in mitochondrial length in hippocampal neurons as described above. Bars indicate mean \pm SEM. $n=71-89$ mitochondria from 6 animals per group. ${ }^{\star *} P<0.01, \star \star \star \star P<0.0001$, by oneway ANOVA with Dunnett's multiple comparisons test. (h) Representative dynamical images of mitochondrial fission and fusion using STED nanoscope. Wild type and 3xTg primary hippocampal neurons were infected with Lv- IncMtDloop at DIV5, cells were labeled with MitoSq-635 $(0.1 \mu \mathrm{M})$ and prepared for tests at DIV14. (i) The effect of restoration of IncMtDloop on cytochrome c oxidase activity using isolated mitochondria of hippocampal neurons as described above. Bars indicate mean \pm SEM. $n=$ 8 per group. ${ }^{* \star \star \star} P<0.0001$, by one-way ANOVA with Dunnett's multiple comparisons test. (j) The mitochondrial oxygen consumption rate (OCR) was assessed in primary hippocampal neurons. 3xTg primary hippocampal neurons were infected with Lv- IncMtDloop or Lv-control at DIV5, cells were prepared for seahorse assay at DIV14. ( $\mathrm{k}$ and I) Restoration of IncMtDloop displayed increased reserved capacity and basal levels of OCR in primary hippocampal neurons as described above. Bars indicate mean \pm SEM. ${ }^{\star} P<0.05,{ }^{*} P<0.01$, by unpaired $t$-test. 
Fig 5

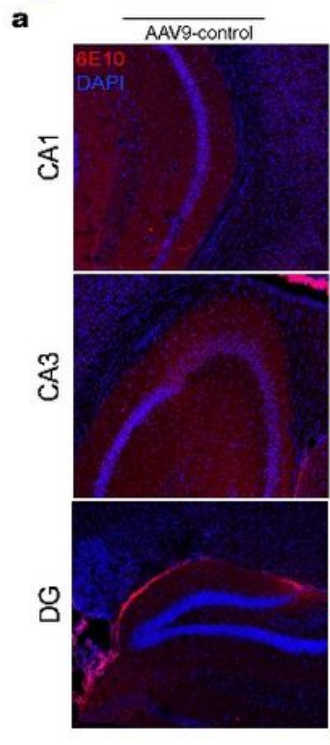

i
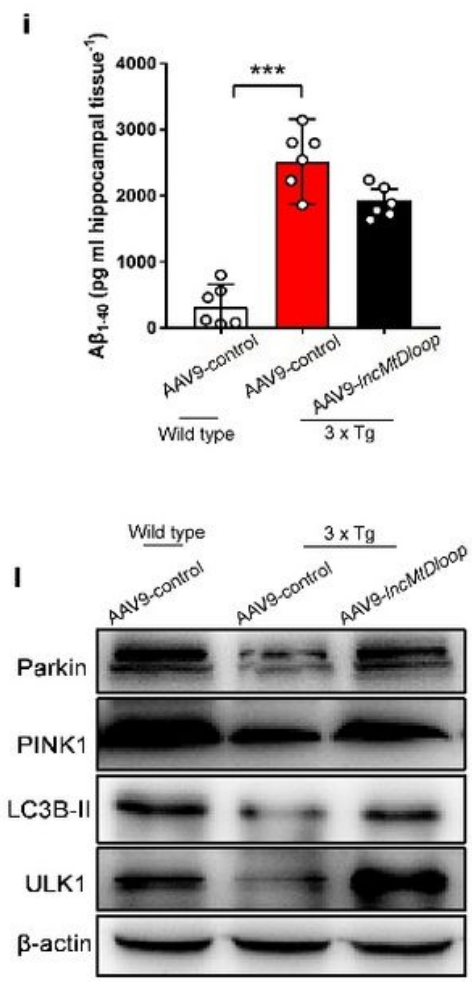

AAV9-control

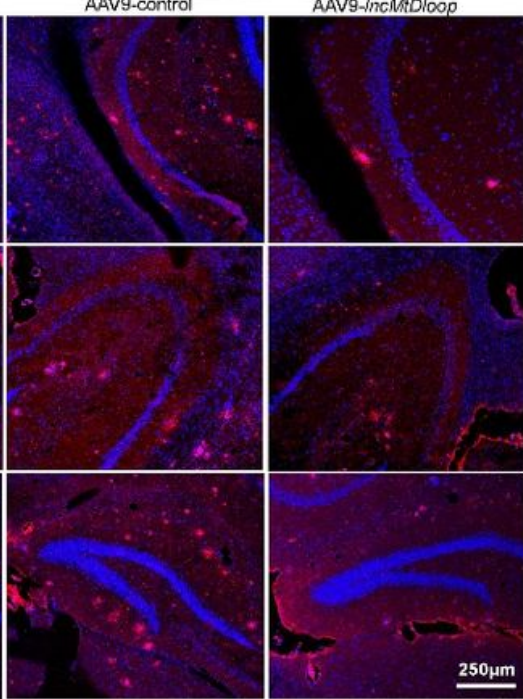

j

Tau-pS202+T205

Tau-pT231

Human Tau

Total Tau

$\beta$-actin
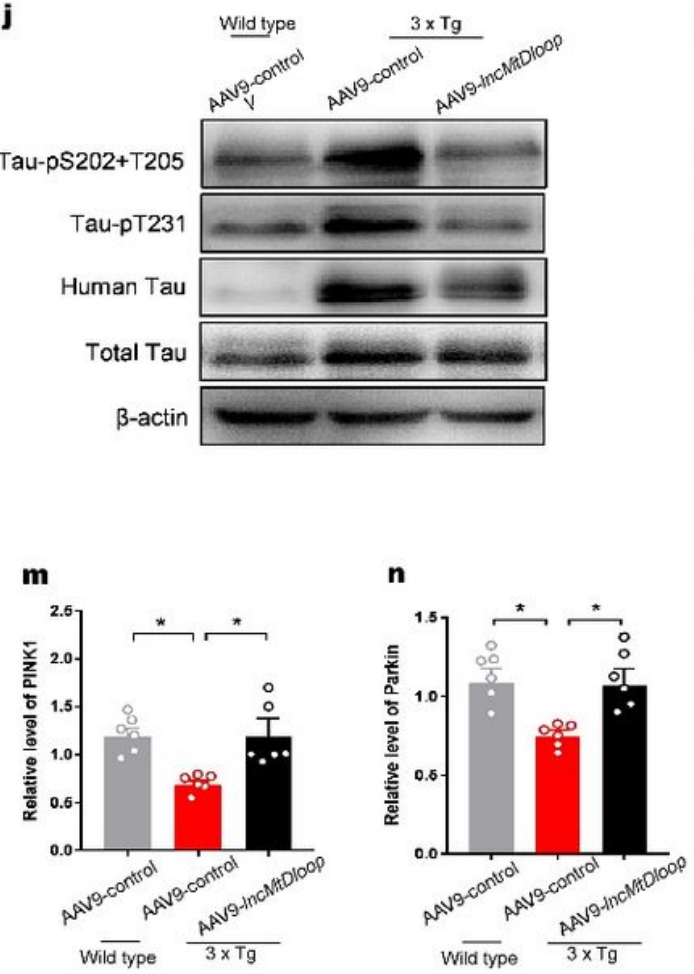

b

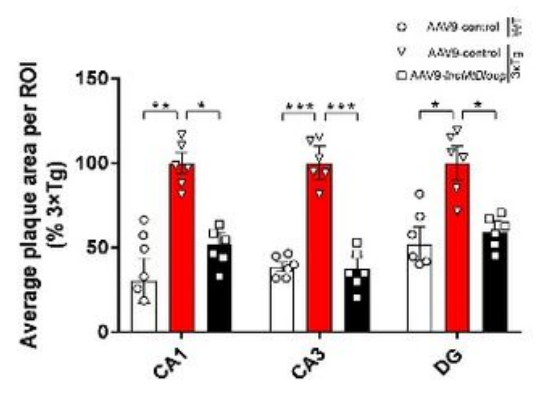

c

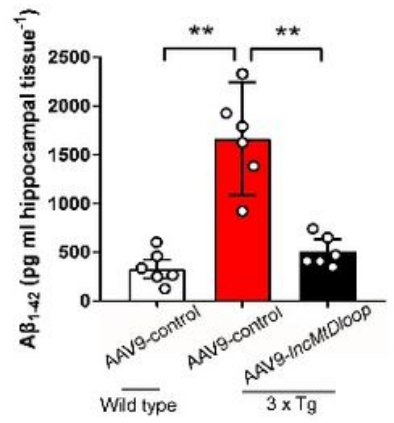

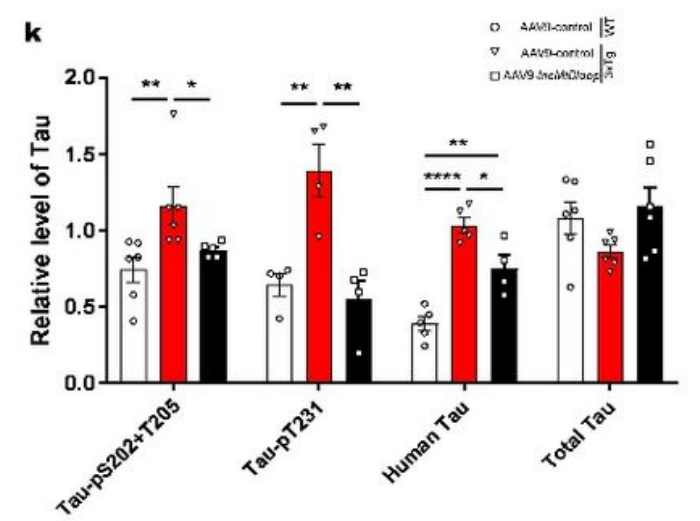

-

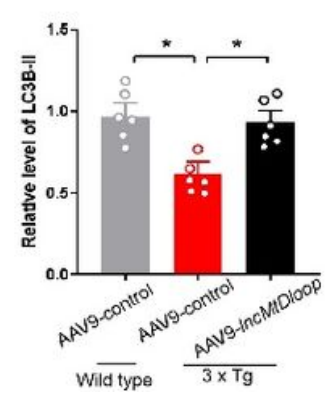

p

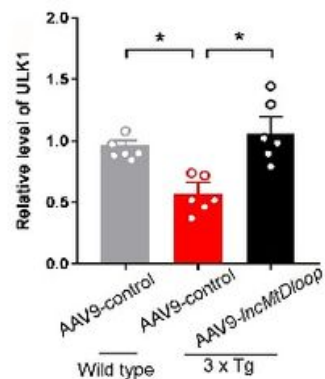

Figure 5

Restoring IncMtDloop expression reverses AD-related pathologies in the brain of the mouse model. (a) Representative IF images showing 6E10 in hippocampus of wild type and 3xTg mice. The viral particles of AAV9-IncMtDloop were microinjected into hippocampus of 11-month-old mice, and 10 $\mu \mathrm{m}$ cryostat slices were prepared and IF with 6E10 antibody at the age of 13 months. (b) Quantification of average A $\beta$ plaque area and fluorescence intensity of $6 \mathrm{E} 10$ in CA1, CA3 and DG of wild type and 3xTg mice as described above. Bars indicate mean \pm SEM. $\mathrm{n}=6$ mice per group. ${ }^{\star \star} P<0.01, * \star \star P<0.001$, by one-way ANOVA with Tukey's multiple comparisons test. (c and d) ELISA analysis showing changes in levels of $A \beta 42$ and $A \beta 40$ in hippocampal tissues of wild type and 3xTg mice as described above. Bars indicate mean \pm SEM. $\mathrm{n}=6$ mice per group. ${ }^{* *} P<0.01,{ }^{* \star *} P<0.001$ by one-way ANOVA with Dunnett's multiple 
comparisons test. (e) Western blot results of AT8, AT180, human tau and total tau in hippocampus of 12month-old wild type and 3xTg mice w/o overexpressing IncMtDloop. (f) Relative intensities of AT8, AT180, human tau and total tau signals illustrated in e). Bars indicate mean \pm SEM. $\mathrm{n}=6$ mice per group. ${ }^{\star} P<0.05,{ }^{* \star} P<0.01,{ }^{\star \star \star \star} P<0.0001$, by one-way ANOVA with Dunnett's multiple comparisons test. (g) Western blot results of parkin, PINK1, LC3B-II and ULK1 showing changes in the event of mitophagy. Proteins were extracted from hippocampus of 12-month-old wild type and 3xTg mice w/o overexpressing IncMtDloop. (h-k) Relative levels of parkin, PINK1, LC3B-II and ULK1 signals illustrated in A). Bars indicate mean \pm SEM. $\mathrm{n}=6$ animals per group. ${ }^{\star} P<0.05$, by one-way ANOVA with Dunnett's multiple comparisons test. 
Fig 6

a AVV stereotaxic

injection into CA1

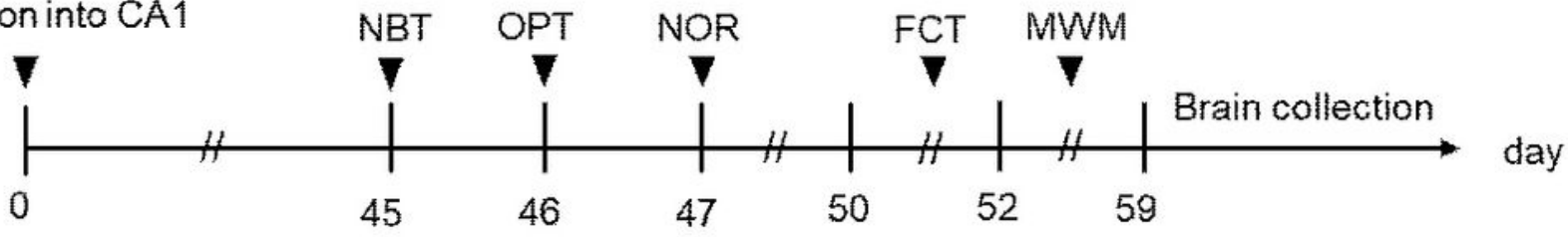

b
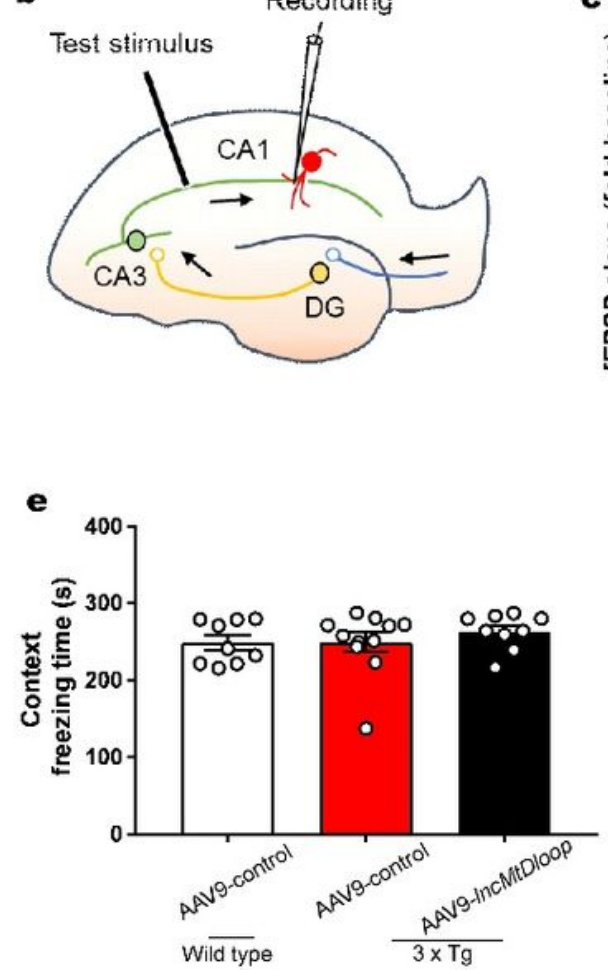

h

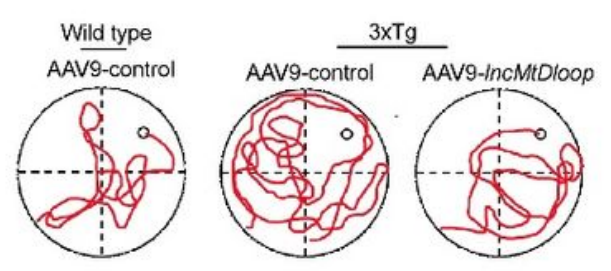

c
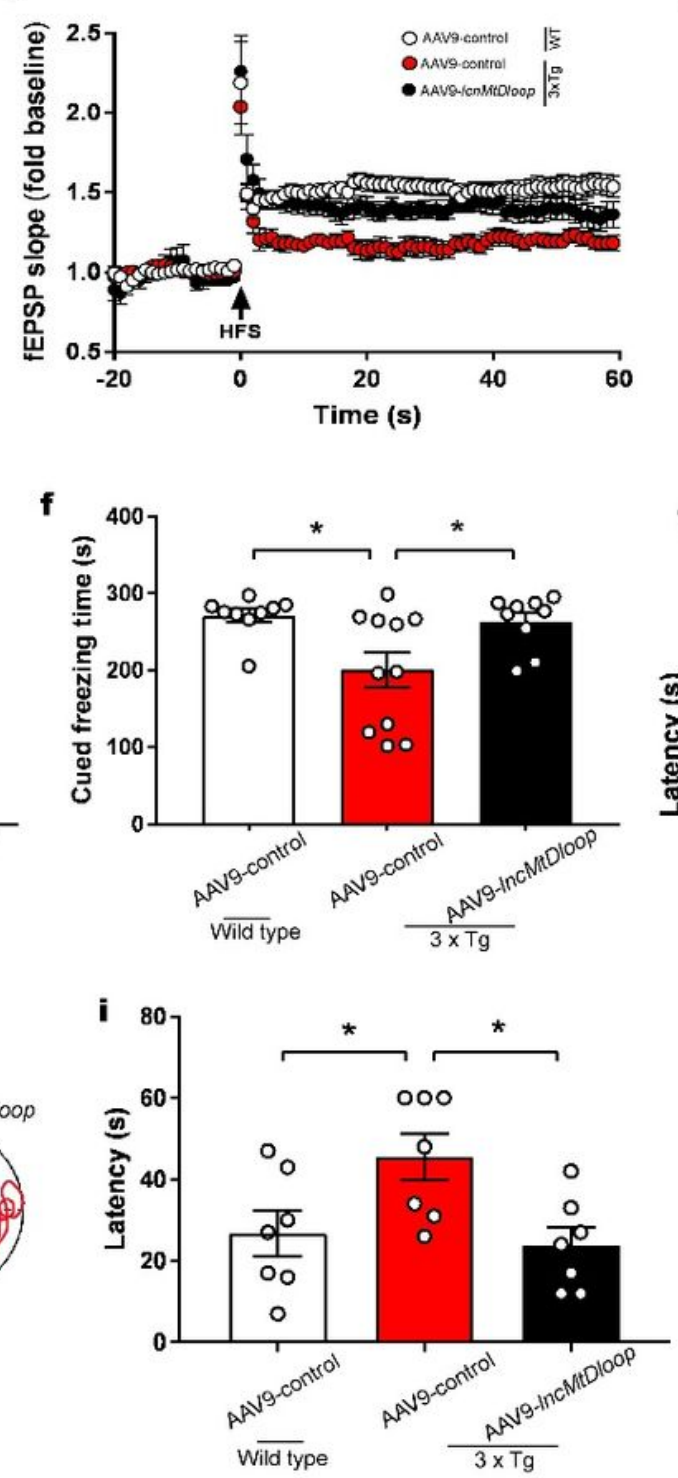

d
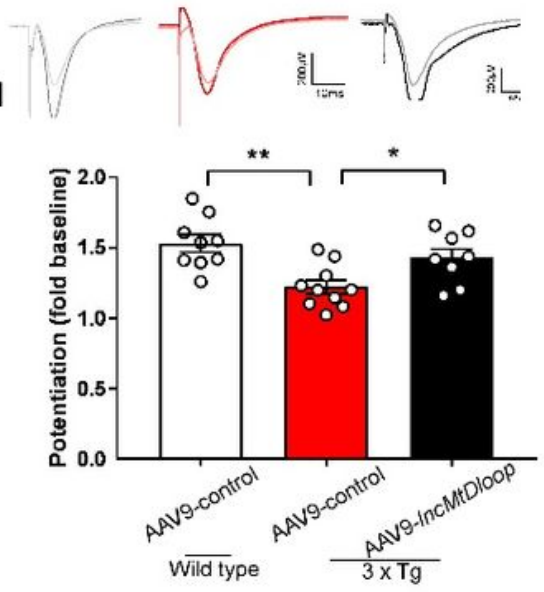

$\mathbf{g}$
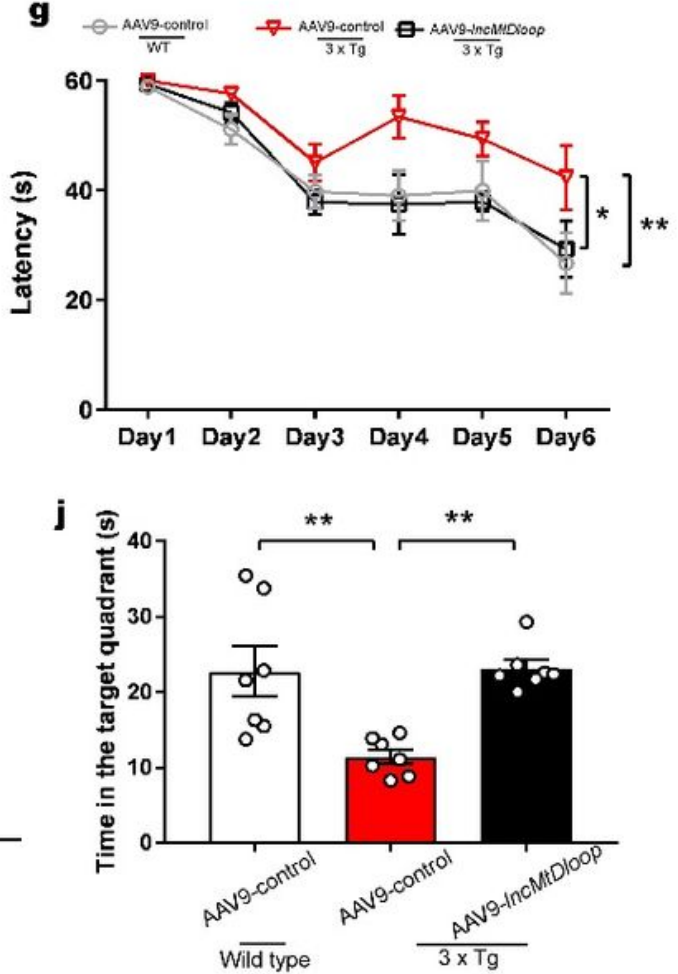

Figure 6

Restoring IncMtDloop reverses deficits in synaptic plasticity and cognitive behaviors of the mouse model. (a) Schematic illustrating a timeline of performing synaptic function and behavior tests. (b) Schematic illustrating the location of recording and stimulating electrodes of synaptic transmission with the Schaffer collateral pathway. (c) Field excitatory postsynaptic potentials (fEPSPs) evoked by stimulation of Schaffer collateral pathway during LTP experiment from acute hippocampal slices from wild type and 
3xTg mice w/o overexpressing IncMtDloop. Bars indicate mean \pm SEM. $n$ (brain slice) $=8-10$ per group from 7 animals per genotype. (d) Representative traces and graphs for electrophysiological recordings with acute hippocampal slices from each group (top). Quantification of average fEPSP from the last $10 \mathrm{~min}$ of the time course from $(C)$. Bars indicate mean \pm SEM. $n$ (brain slice) $=8-10$ per group from 7 animals per genotype. ${ }^{*} P<0.05,{ }^{*} P<0.01$, by one-way ANOVA with Dunnett's multiple comparisons test (bottom). (e-f) Contextual fear conditioning test and cued fear conditioning test were performed in mice as described above. Bars indicate mean \pm SEM. $n=9-11$ animals per group. ${ }^{*} \mathrm{P}<0.05$, by one-way ANOVA with Dunnett's multiple comparisons test. (g) Escape latencies of each group in the MWM during a 5-day training period. Bars indicate mean \pm SEM. $\mathrm{n}=7$ animals per group. ${ }^{*} P<0.05, \star \star * P<0.01$, by two-way ANOVA with Tukey's multiple comparisons test. (h) Escape traces to the hidden platform of each group in the MWM at day 6. (i) Latencies to the target platform of each group in the MWM at day 6 . Bars indicate mean \pm SEM. $\mathrm{n}=7$ animals per group. ${ }^{*} P<0.05$, by one-way ANOVA with Dunnett's multiple comparisons test. (j) The time exploring in the target quadrant in the MWM at day 6. Bars indicate mean \pm SEM. $n=7$ animals per group. ${ }^{*} P<0.05$, by one-way ANOVA with Dunnett's multiple comparisons test.

\section{Supplementary Files}

This is a list of supplementary files associated with this preprint. Click to download.

- SupplementaryFiguresLiJ.pdf

- SupplementaryTable1.pdf

- SupplementaryTable2.xlsx

- SupplementaryVideo3.avi

- SupplementaryVideo4.avi

- SupplementaryVideo1.avi

- SupplementaryVideo2.avi 